This is the post peer-review accepted manuscript of:

Guerra, A., Guidi, F., \& Dardari, D. (2018). Single-Anchor Localization and Orientation Performance Limits Using Massive Arrays: MIMOvs.Beamforming. IEEE Transactions on Wireless Communications, 17(8), 5241-5255. https://doi.org/10.1109/TWC.2018.2840136

The published version is available online at: https://doi.org/10.1109/TWC.2018.2840136

C 2018 IEEE. Personal use of this material is permitted. Permission from IEEE must be obtained for all other uses, in any current or future media, including reprinting/republishing this material for advertising or promotional purposes, creating new collective works, for resale or redistribution to servers or lists, or reuse of any copyrighted component of this work in other works 


\title{
Single-Anchor Localization and Orientation Performance Limits using Massive Arrays: MIMO vs. Beamforming
}

\author{
Anna Guerra, Member, IEEE, Francesco Guidi, Member, IEEE, Davide Dardari Senior Member, IEEE
}

\begin{abstract}
In next generation of cellular networks, it is desirable to use single access points both for communication and localization. This could be made possible thanks to the combination of femtocells, mm-wave communications and massive antenna arrays and would overcome the problem of having an over-sized infrastructure for positioning which is, nowadays, the bottleneck for the widespread diffusion of indoor localization systems. In this context, our paper aims at investigating the localization and orientation performance limits employing massive arrays both at the access point (AP) and mobile side. To this end, we first asymptotically demonstrate the tightness of the CramérRao bound (CRB) in the massive array regime and that the effect of multipath can be made negligible even for practical values of SNR levels. Successively, we propose a comparison between two different transmitter configurations, namely MIMO where orthogonal waveforms are sent, and beamforming, which takes advantage of highly correlated waveforms and directive array patterns. We also consider random weighting as a trade-off between the diversity gain of MIMO and the high directivity guaranteed by the beamforming. CRB results show the interplay between diversity and beamforming gain as well as the benefits achievable by varying the number of antennas in terms of localization accuracy and multipath mitigation.
\end{abstract}

Index Terms-Position and Orientation Error Bound, Massive array, Asymptotic Fisher Information Analysis, Direct Localization.

\section{INTRODUCTION}

Different technologies have been proposed for fifth generation $(5 \mathrm{G})$ and beyond $5 \mathrm{G}(\mathrm{B} 5 \mathrm{G})$ wireless communication networks to meet the ever-increasing traffic demand [1]. Among them, large-scale antenna arrays at base stations (BSs) or access points (APs) allow to smartly direct the power flux towards intended users, and millimeter-wave (mm-wave) communication takes advantage of a less crowded and larger spectrum [2], [3]. Apart from communication-oriented applications, it is expected that, thanks to these technologies, the possibility of accurately locating personal devices, even in Global Positioning System (GPS)-denied environments, will undergo a considerable improvement in terms of performance [4]-[6]. In this context, the same AP used for communication can be

Anna Guerra, Francesco Guidi and Davide Dardari are with the Dipartimento di Ingegneria dell'Energia Elettrica e dell'Informazione "Guglielmo Marconi" - DEI, University of Bologna, via Venezia 52, 47521 Cesena, ITALY. (e-mail: anna.guerra3@unibo.it, f.guidi@unibo.it, davide.dardari@unibo.it).

The work has been partially funded by the European Commission, under Horizon 2020 project XCYCLE (grant nr. 635975), IF-EF Marie SklodowskaCurie project MAPS (grant $\mathrm{nr}$. 659067) and IF-GF Marie Sklodowska-Curie project AirSens (grant nr. 793581). also exploited as a single-anchor node, i.e., a node whose position is a-priori known from an estimation perspective, able to infer the positions of the mobiles with an unprecedented localization accuracy. Furthermore, the adoption of arrays at the transmitter (Tx) (mobile) and receiver (Rx) (AP) will allow to estimate the user's orientation with a precision higher than that provided by compass and gyroscopes, enabling applications such as augmented reality or simultaneous localization and mapping (SLAM) [7]-[10].

Contrarily to traditional localization scenarios, where dedicated multiple anchor nodes are necessary to allow classic triangulation/multilateration techniques [11], the possibility to centralize both communication and localization capabilities in a single multi-antenna AP can be envisioned with the advantage of drastically decreasing the overall system complexity and cost. Moreover, when moving up in frequencies, not only APs but also mobiles can adopt massive arrays, thus increasing even more the performance given the potential huge set of measurements available [12], [13]. In such a scenario, it becomes of great interest to understand the fundamental limits on localization error using a single anchor node and exploiting the potentialities of massive arrays.

Concerning the ultimate localization performance evaluation, a rich literature has been produced for the analysis of multiple anchor systems (see for example [14]-[16]). In these works, the authors usually consider a 2D multipleanchor localization scenario where measurements come from multiple widely distributed single-antenna anchors. A common approach followed in the literature is that of adopting a 2-step method in which the position is estimated from time-of-arrival (TOA), angle-of-arrival (AOA) and angle-of-departure (AOD) instead of being directly inferred from the received signals [5], [14], [15]. These approaches do not ensure the derived bounds represent a fundamental limit over any possible position estimator; in fact, as stated by the information processing inequality in information theory [17], estimation performed by processing intermediate parameters (e.g., TOA-AOA-AOD) is in general sub-optimum.

In addition, in the current state-of-the-art, there are neither studies comparing different array configurations and architectures (e.g., multiple-input multiple-output (MIMO), beamforming) nor asymptotic investigations in the massive array regime to study the effect of multipath and the tightness of the CRB. Recent results [5] and [16] exploit the typical channel sparsity at mm-wave and the higher angular resolution using large arrays to resolve and mitigate multipath components, 
even though such results cannot be extended in general to lower frequencies.

In this paper, we adopt a direct single-anchor localization approach in which the Tx position and orientation are directly inferred from the received signals [12], [18]. Differently from [5], [16], [19], the aim of our work is to compare different massive array signal processing schemes at the Tx, i.e. beamforming and MIMO, and to provide a useful tool for system design in terms of position and orientation performance. Throughout this analysis, the trade-off between the signal-to-noise ratio (SNR) enhancement obtained via beamforming (where the transmitted signals are fully correlated to form a main directive beam) and the diversity gain obtained using orthogonal signals at Tx side (e.g., MIMO) is highlighted. The idea of using random weighting, i.e., arrays in which beamforming weights are randomly chosen, is also put forth and its performance is compared to that of classical beamforming and MIMO solutions. Beamforming non-idealities [20], [21] and phase synchronization uncertainty [16], [22] are also taken into account in the analysis. In our previous works [19], [23], some preliminary results on positioning accuracy considering only beamforming strategies have been presented, whereas the MIMO configuration and the multipath were not considered.

Then, contrarily to several papers where authors assume to work in high SNR regime with a lower number of array antennas (for example, [5], [9], [14]-[16]), here we demonstrate through an ambiguity function (AF) analysis [24], the tightness of the CRB independently from the array configuration and the operating SNR, provided that a large number of antennas is involved (massive-array regime). This turns to be an important result derived using massive arrays for localization purposes. Finally, using the same asymptotic analysis, we demonstrate that the use of massive arrays drastically reduces the interference due to multipath components (MPCs) in a similar way as interference acts in massive MIMO communications [25].

To sum up, the main contributions of this work can be summarized as follows:

- Derivation of the theoretical performance limits on the localization and orientation error for different array configurations in a single-anchor scenario;

- Proposal of a signal model valid for any antenna array geometry, configuration (i.e., MIMO, phased, timed arrays), and frequency band. As a case study, in the numerical results we focus on mm-wave massive arrays due to their attractiveness in 5G and B5G applications;

- Introduction of a low-complexity random weighting approach, i.e., randomly chosen beamforming weights, and analysis of its performance compared to that of classical beamforming and MIMO solutions;

- Investigation of the $\mathrm{CRB}$ tightness in massive array regime (i.e., massively increasing the number of antennas) for any SNR condition;

- Analysis of the trade-off between SNR enhancement obtained via beamforming and diversity gain of MIMO considering the impact of different types of uncertainties as, for example, the MPCs, beamforming weights and synchronization residual mismatch;

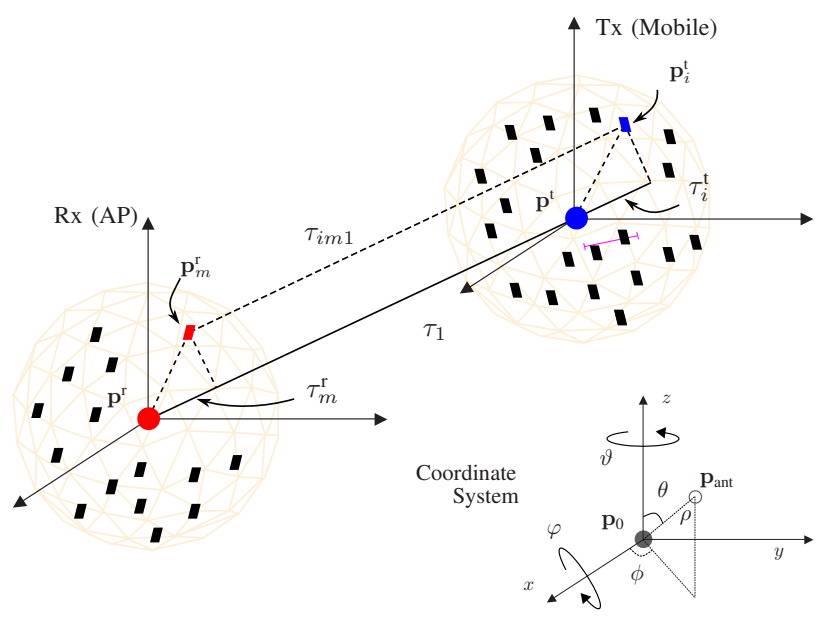

Fig. 1. Array geometric configuration in free-space.

- Demonstration that in the massive array regime the effect of multipath can be made negligible on average regardless the SNR condition.

The rest of the paper is organized as follows. Sec. II describes the geometry of the localization system. Sec. III introduces the signal model taking into account different array configurations. In Sec. IV the localization performance limits are derived. Sec. V analyzes the asymptotic conditions for which the CRB can be considered a tight bound. Sec. VI compact formulas for an ideal free-space case are derived. The multipath impact on localization performance is investigated in Sec. VII. Finally, Sec. VIII presents the results and Sec. IX concludes the work.

Notation: Lower case and capital letters in bold denote vectors and matrices, respectively. The subscripts $[\cdot]^{\mathrm{T}},[\cdot]^{*}$ and $[\cdot]^{\mathrm{H}}$ indicate the transpose, the conjugate and the Hermitian operators. $\|\cdot\|_{2}$ is the Euclidean norm, $\mathbf{A} \succeq \mathbf{B}$ indicates that the matrix $\mathbf{A}-\mathbf{B}$ is non-negative definite, and diag (.) represents the diagonal operator. The subscripts $(\cdot)^{\mathrm{t}}$ and $(\cdot)^{\mathrm{r}}$ refer to quantities related to the Tx and Rx array, respectively. $(\cdot)^{\mathrm{FS}}$ indicates the free-space scenario. $\mathcal{F}(\cdot)$ denotes the Fourier transform operation, $\mathcal{U}(a, b)$ a uniform distribution in the interval $[a, b]$, and $\mathcal{C N}\left(\mu, \sigma^{2}\right)$ a circular complex symmetric Gaussian distribution with mean $\mu$ and variance $\sigma^{2}$.

\section{Antenna Array Geometric Configuration}

We consider a 3D localization scenario, as the one reported in Fig. 1, consisting of a single AP acting as reference Rx node equipped with a $N_{\mathrm{rx}}$-antenna array, and a Tx mobile terminal with a $N_{\mathrm{tx}}$-antenna array. In the rest of the manuscript, the different antenna array configurations refer to the Tx array, whereas the ultimate localization performance limits are evaluated at the $\mathrm{Rx}$ side.

The aim of the localization is to directly infer the position of the Tx centroid $\mathbf{p}^{\mathrm{t}}=\left[x_{0}^{\mathrm{t}}, y_{0}^{\mathrm{t}}, z_{0}^{\mathrm{t}}\right]^{\mathrm{T}}=[x, y, z]^{\mathrm{T}}$ and its orientation $\boldsymbol{\vartheta}^{\mathrm{t}}=\left[\vartheta^{\mathrm{t}}, \varphi^{\mathrm{t}}\right]^{\mathrm{T}}$ when the $\mathrm{Rx}$ centroid position $\mathbf{p}^{\mathrm{r}}=\left[x_{0}^{\mathrm{r}}, y_{0}^{\mathrm{r}}, z_{0}^{\mathrm{r}}\right]^{\mathrm{T}}=[0,0,0]^{\mathrm{T}}$ and its orientation $\vartheta^{\mathrm{r}}=\left[\vartheta^{\mathrm{r}}, \varphi^{\mathrm{r}}\right]^{\mathrm{T}}$ are known. ${ }^{1}$

\footnotetext{
${ }^{1}$ Without loss of generality, the $\mathrm{Rx}$ is assumed located at the origin of the coordinates system.
} 
Throughout the paper, we designate with:

- Fixed orientation: the specific geometric configuration with the Tx and the Rx parallel to each other (i.e., $\left.\boldsymbol{\vartheta}^{\mathrm{t}}=\boldsymbol{\vartheta}^{\mathrm{r}}=[0,0]^{\mathrm{T}}\right)$;

- Averaged orientation: the geometric configuration in which a different 3D Rx array orientation (i.e., $\vartheta^{\mathrm{r}}=$ $\left.\left[\vartheta^{\mathrm{r}}, \varphi^{\mathrm{r}}\right]^{\mathrm{T}}\right)$ is generated at each Monte Carlo iteration, and the $\mathrm{CRB}$ is computed as the average over all the partial $\mathrm{CRB}$ results computed at each cycle. In this case, the Tx orientation is fixed to $\vartheta^{\mathrm{t}}=[0,0]^{\mathrm{T}}$.

With reference to Fig. $1, \mathbf{p}_{i}^{\mathrm{t}}\left(\boldsymbol{\vartheta}^{\mathrm{t}}\right)=\left[x_{i}^{\mathrm{t}}, y_{i}^{\mathrm{t}}, z_{i}^{\mathrm{t}}\right]^{\mathrm{T}}$ indicates the position of the $i$ th transmitting antenna relative to the Tx geometric center and dependent on the Tx orientation, and $\mathbf{p}_{m}^{\mathrm{r}}\left(\boldsymbol{\vartheta}^{\mathrm{r}}\right)=\left[x_{m}^{\mathrm{r}}, y_{m}^{\mathrm{r}}, z_{m}^{\mathrm{r}}\right]^{\mathrm{T}}$ the position of the $m$ th receiving antenna relative to the $\mathrm{Rx}$ geometric center. Considering spherical coordinates, we have $\mathbf{p}_{n}=\rho_{n} \mathbf{R}(\boldsymbol{\vartheta}) \mathbf{d}^{\mathrm{T}}\left(\boldsymbol{\theta}_{n}\right)$, with $n=1, \ldots, N$ being the generic antenna index and $N$ the total number of array antennas either at the $\mathrm{Tx}$ or $\mathrm{Rx}$ side. The direction cosine is

$$
\mathbf{d}(\boldsymbol{\theta})=[\sin (\theta) \cos (\phi), \sin (\theta) \sin (\phi), \cos (\theta)]
$$

while $\rho_{n}=\left\|\mathbf{p}_{n}(\boldsymbol{\vartheta})-\mathbf{p}\right\|_{2}$ and $\boldsymbol{\theta}_{n}=\left[\theta_{n}, \phi_{n}\right]^{\mathrm{T}}$ are the distance and angle between the considered antenna from the correspondent array centroid (whose position is here indicated as $\mathbf{p}$ ). The rotational matrix $\mathbf{R}(\boldsymbol{\vartheta})$ is given by $\mathbf{R}(\boldsymbol{\vartheta})=\mathbf{R}_{z}(\varphi) \mathbf{R}_{x}(\vartheta)$, where $\mathbf{R}_{z}(\varphi)$ and $\mathbf{R}_{x}(\vartheta)$ define the counter-clockwise rotation around the $z$-axis and the clockwise rotation around the $x$-axis, respectively. Finally $\boldsymbol{\theta}_{1}=\left[\theta_{1}, \phi_{1}\right]^{\mathrm{T}}$ designates the angle of incidence between arrays centroids (direct path) and $\boldsymbol{\theta}_{0}=\left[\theta_{0}, \phi_{0}\right]^{\mathrm{T}}$ represents the intended steering direction when applied.

The diameter $D$ of the transmitting and receiving arrays is assumed much smaller than the inter-array distance $d=$ $\left\|\mathbf{p}^{\mathrm{r}}-\mathbf{p}^{\mathrm{t}}\right\|_{2}$, i.e., $D \ll d$. Note that this hypothesis is especially verified at mm-wave where the array dimensions are very small thanks to the reduced wavelength [20]. Moreover the arrays are supposed to be sufficiently far from the surrounding scatterers, thus obtaining identical angles of incidence for both direct and MPCs at each antenna element.

In our scenario, we take $L$ MPCs into consideration as nuisance parameters in the localization process, i.e., no relationship between MPCs characteristics and position is available. Thus, we assume that the first path always experiences a line-of-sight (LOS) propagation condition. For what the MPCs parameters are concerned, we follow the same notation introduced in [16]. In particular, let $\boldsymbol{\theta}_{l}^{\mathrm{t}}=$ $\left[\theta_{l}^{\mathrm{t}}, \phi_{l}^{\mathrm{t}}\right]^{\mathrm{T}}=\left[\theta_{1}+\Delta \theta_{l}^{\mathrm{t}}, \phi_{1}+\Delta \phi_{l}^{\mathrm{t}}\right]^{\mathrm{T}}$ and $\boldsymbol{\theta}_{l}^{\mathrm{r}}=\left[\theta_{l}^{\mathrm{r}}, \phi_{l}^{\mathrm{r}}\right]^{\mathrm{T}}=$ $\left[\theta_{1}+\Delta \theta_{l}^{\mathrm{r}}, \phi_{1}+\Delta \phi_{l}^{\mathrm{r}}\right]^{\mathrm{T}}$, with $l=1,2, \ldots, L$, indicate the angles of departure from the transmitting array and of incidence at the $\mathrm{Rx}$ side of the $l$ th path, respectively. The angular biases $\left[\Delta \theta_{l}^{\mathrm{t}}, \Delta \phi_{l}^{\mathrm{t}}\right]^{\mathrm{T}}$ and $\left[\Delta \theta_{l}^{\mathrm{r}}, \Delta \phi_{l}^{\mathrm{r}}\right]^{\mathrm{T}}$ are the displacement with respect to the direct path at the Tx and Rx side. Note that for the direct path it holds $\left[\Delta \theta_{1}^{\mathrm{t}}, \Delta \phi_{1}^{\mathrm{t}}\right]^{\mathrm{T}}=\left[\Delta \theta_{1}^{\mathrm{r}}, \Delta \phi_{1}^{\mathrm{r}}\right]^{\mathrm{T}}=[0,0]^{\mathrm{T}}$.

Let $\tau_{1} \triangleq\left\|\mathbf{p}^{\mathrm{r}}-\mathbf{p}^{\mathrm{t}}\right\|_{2} / c=d / c$ and $\tau_{i m 1} \triangleq\left\|\mathbf{p}_{m}^{\mathrm{r}}-\mathbf{p}_{i}^{\mathrm{t}}\right\|_{2} / c$ being the propagation delay related to the direct path between the transmitting and receiving centroids and between the $i$ th and $m$ th antenna, respectively, where $c$ is the speed of light.
Considering the multipath, the $l$ th propagation delay between array centroids is defined as $\tau_{l}=\tau_{1}+\Delta \tau_{l}$ where $\Delta \tau_{l}$ is the non-negative delay bias of the $l$ th path, with $\Delta \tau_{1}=0$. According to the geometric assumption previously described, the TOA and amplitude between each couple of transmitting and receiving antennas can be expressed using the following two approximations [16]

$$
\tau_{i m l} \approx \tau_{l}+\tau_{i}^{\mathrm{t}}\left(\boldsymbol{\theta}_{l}^{\mathrm{t}}, \boldsymbol{\vartheta}^{\mathrm{t}}\right)-\tau_{m}^{\mathrm{r}}\left(\boldsymbol{\theta}_{l}^{\mathrm{r}}, \boldsymbol{\vartheta}^{\mathrm{r}}\right), \quad a_{i m l} \approx a_{l}
$$

where $a_{i m l}$ is the amplitude of the $l$ th path between the $m$ th receiving and the $i$ th transmitting antenna, and $\tau_{m}^{\mathrm{r}}\left(\boldsymbol{\theta}_{l}^{\mathrm{r}}, \boldsymbol{\vartheta}^{\mathrm{r}}\right)$ and $\tau_{i}^{\mathrm{t}}\left(\boldsymbol{\theta}_{l}^{\mathrm{t}}, \boldsymbol{\vartheta}^{\mathrm{t}}\right)$ are the Rx and Tx inter-antenna propagation delays, respectively, that are shown in Fig. 1 and are defined as

$$
\tau_{n}\left(\boldsymbol{\theta}_{l}, \boldsymbol{\vartheta}\right)=\frac{1}{c} \mathbf{d}\left(\boldsymbol{\theta}_{l}\right) \mathbf{p}_{n}(\boldsymbol{\vartheta}), \quad n=1, \ldots, N
$$

\section{A. Special Case: Planar Array Geometry}

Planar arrays appear to be the most suitable when considering the integration of massive arrays in portable devices or in small spaces [13]. For this reason, in addition to the general analysis valid for any geometry (i.e., any antennas spatial deployment), some compact specialized equations will be derived in Sec. VI for squared arrays of area $A=d_{\text {ant }}^{2} N$ with the antennas equally spaced of $d_{\text {ant }}$. In this case, if the array lies on the $X Z$-plane, the antenna coordinates are

$$
\mathbf{p}_{n}(\boldsymbol{\vartheta})=\left[x_{n}, 0, z_{n}\right]^{\mathrm{T}}=\mathbf{R}(\boldsymbol{\vartheta})\left[n_{x} d_{\mathrm{ant}}, 0, n_{z} d_{\mathrm{ant}}\right]^{\mathrm{T}}
$$

where $n_{x}=n_{z}=-\frac{\sqrt{N}-1}{2},-\frac{\sqrt{N}-3}{2}, \ldots, \frac{\sqrt{N}-1}{2}$ are the antenna indexes along the $x$ and $z$ axis, respectively.

\section{Antenna Array Schemes and Signal Model}

In this section, different types of antenna array schemes are analyzed starting from a unified signal model with the purpose to highlight their impact on localization. In the rest of the manuscript, we refer to the following definitions:

- Beamforming: At the Tx side, the signals at each antenna branch are weighted in order to form a beam pointing towards the $\mathrm{Rx}$ node. This means that the same signal passes through a network of time delay line (TDL) and/or phase shifters (PSs) whose weights permit to align all the signals in the desired direction, as in Fig. 2-(a,b). Moreover, we suppose that the beamsteering at the Tx is done to exactly point towards the Rx;

- MIMO: The transmitted signals are orthogonal to each other (see Fig. 2-(c)) and, therefore, there is not the formation of a main beam at the Tx side. Nevertheless, the orthogonality is kept over the channel and the Rx is able to distinguish among the transmitted waveforms and to retrieve location-related information;

- Random Weighting: The Tx array configuration is constituted of only PSs whose weights are randomly chosen, as shown in Fig. 2-(d). Hence, this approach maintains the same low-complexity of beamforming but without beam formation.

Consequently, the difference between beamforming and MIMO regards only the transmitted signals. Contrarily, at the 
(a) Phased Array

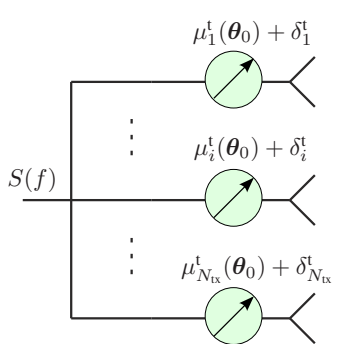

(b) Timed Array

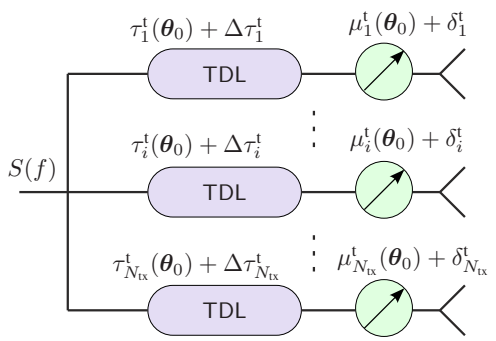

(c) MIMO

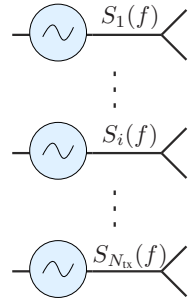

(d) Random Weighting

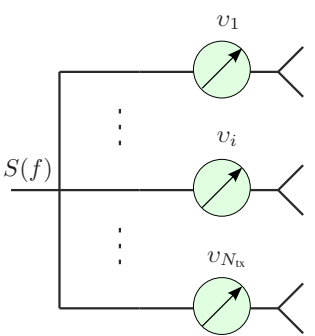

Fig. 2. From the left to the right: Phased, timed, MIMO and random weighting array schemes.

$\mathrm{Rx}$, which is the anchor node for localization, the CRB is computed by considering the signals collected at each antenna branch regardless the Rx architecture itself.

\section{A. Transmitted Signal Model}

The transmitted signal at the $i$ th antenna is denoted with $g_{i}(t)=\Re\left\{s_{i}(t) e^{j 2 \pi f_{\mathrm{c}} t}\right\}$, where $s_{i}(t)$ represents the equivalent low-pass signal and $f_{\mathrm{c}}$ the carrier frequency. We consider a constraint on the total transmitted energy $E_{\text {tot }}$ which is uniformly allocated among antennas, where $E=E_{\text {tot }} / N_{\mathrm{tx}}=$ $\int\left|s_{i}(t)\right|^{2} d t, i=1,2, \ldots, N_{\mathrm{tx}}$, represents the normalized energy at each antenna element. We introduce the Fourier transform of $s_{i}(t)$ as $S_{i}(f)=\mathcal{F}\left\{s_{i}(t)\right\}$, with $\mathcal{F}\{\cdot\}$ denoting the Fourier transform operation in a suitable observation interval containing the signal support. For further convenience, the vector $\mathbf{s}(f)=\left[S_{1}(f), \ldots, S_{N_{\mathrm{tx}}}(f)\right]^{\mathrm{T}}$ contains all the baseband transmitted signals. In the following, the signal model for each array configuration will be further detailed with reference to Fig. 2.

1) Timed and phased arrays: In multi-antenna systems, beamforming is obtained by applying a progressive time delay at each array element so that the emitted signals result summed up coherently towards the intended steering direction. Considering the signal bandwidth $W$, when the condition $W \ll f_{\mathrm{c}}$ holds, this process can be well approximated using only PSs (the corresponding array structure is denoted as phased array). On the contrary, when $W \approx f_{\mathrm{c}}$, phase shifts are no longer sufficient to align all the signals. As a consequence, to avoid beamsteering ${ }^{2}$ degradation (e.g., squinting effect), timed arrays consisting of PSs and TDLs must be introduced.

Given these array schemes, the transmitted signal is the same for all transmitting antennas, i.e., $s_{i}(t)=s(t)=$ $\sqrt{E} p(t) \forall i=1, \ldots, N_{\mathrm{tx}}$, with $p(t)$ being the unitary energy normalized version of $s(t)$, and beamforming weights are applied to each branch of the array to focus the power flux in a precise direction in space. Specifically, when no quantization errors are present in the weights, the beamforming matrix is

$$
\mathbf{B}\left(f, \boldsymbol{\theta}_{0}\right)=\operatorname{diag}\left(\omega_{1}, \omega_{2}, \ldots, \omega_{i}, \ldots \omega_{N_{\mathrm{tx}}}\right) .
$$

The $i$ th beamforming weight is $\omega_{i}=b_{i}(f) b_{i}^{\mathrm{c}}$, with $b_{i}(f)=$ $e^{j 2 \pi f \tau_{i}^{\mathrm{t}}\left(\boldsymbol{\theta}_{0}\right)}$ and $b_{i}^{\mathbf{c}}=e^{j \mu_{i}^{\mathrm{t}}\left(\boldsymbol{\theta}_{0}\right)}$, where $\mu_{i}^{\mathrm{t}}\left(\boldsymbol{\theta}_{0}\right)=2 \pi f_{\mathrm{c}} \tau_{i}^{\mathrm{t}}\left(\boldsymbol{\theta}_{0}\right)$ and $\tau_{i}^{\mathrm{t}}\left(\boldsymbol{\theta}_{0}\right)$ are the transmitting steering phase and delay related to the $i$ th PS and TDL of the array, respectively. The main

\footnotetext{
${ }^{2}$ The terms beamsteering and beamforming are here used as synonymous.
}

difference between phased and timed array is the way in which the beamsteering process is performed: in the former, only PSs are present (i.e., $\tau_{i}^{\mathrm{t}}\left(\boldsymbol{\theta}_{0}\right)=0 \forall i=1, \ldots, N_{\mathrm{tx}}$ (refer to Fig. 2-(a)) while, in the latter, TDLs and PSs are both employed to counteract the beamsquinting effect caused by a larger $W / f_{\text {c }}$ ratio (see Fig. 2-(b)). Nevertheless, some technological issues could induce errors in the beamforming vector. Firstly, when digitally controlled PSs are used in place of their high-resolution analog counterparts, the presence of quantization errors has to be accounted for [7]. As shown in [20], where some massive arrays prototypes working in the $\mathrm{X}$ - and V-bands have been proposed, PSs can be realized by simply adopting switches, or by rotating patch antennas. Therefore, continuous phase shifts ranging from $0^{\circ}$ to $360^{\circ}$ are not realizable in practice and the quantization errors generated by the discretization of phases should be taken into account when considering real arrays.

Secondly, synchronization between the Tx and the Rx is required to estimate the position. There are several techniques to accomplish this task [26], [27], with the two-way ranging being one of the most used. Unfortunately due to several factors such as clock drift, a residual synchronization error, affecting both the delay and the phase, is always present and it is accounted by the term $\epsilon^{\mathrm{s}}$ in our model. This mismatch can be also used to model the non-perfect Tx-Rx phase coherence, which is particularly challenging to attain when moving up in frequencies. In the other way round, a significant synchronization error could vanish the advantages of having a coherent processing. In the presence of such non-perfect weights and synchronization error, a matrix accounting for all the non-idealities is introduced in the form

$$
\mathbf{Q}(f)=e^{-j 2 \pi\left(f+f_{\mathrm{c}}\right) \epsilon^{\mathrm{s}}} \operatorname{diag}\left(\varsigma_{1}, \varsigma_{2}, \ldots, \varsigma_{i}, \ldots, \varsigma_{N_{\mathrm{tx}}}\right)
$$

where $\varsigma_{i}$ takes into account the $i$ th beamforming weight quantization error, i.e., $\varsigma_{i}=e^{j\left(2 \pi f \Delta \tau_{i}^{t}+\delta_{i}^{t}\right)}$ with $\delta_{i}^{\mathrm{t}}$ being the phase error and $\Delta \tau_{i}^{\mathrm{t}}$ the TDL error. For further convenience, let indicate with $\tilde{\omega}_{i}=\tilde{b}_{i}(f) \tilde{b}_{i}^{\mathrm{c}}$ the quantized weights, with $\tilde{b}_{i}(f)=e^{j 2 \pi f\left(\tau_{i}^{\mathrm{t}}\left(\boldsymbol{\theta}_{0}\right)+\Delta \tau_{i}^{t}\right)}$ and $\tilde{b}_{i}^{\mathrm{c}}=e^{j\left(\mu_{i}^{\mathrm{t}}\left(\boldsymbol{\theta}_{0}\right)+\delta_{i}^{\mathrm{t}}\right)}$. After the beamforming process, the signal at each antenna element can be written as $\mathbf{Q}(f) \mathbf{B}\left(f, \boldsymbol{\theta}_{0}\right) \mathbf{s}(f)$.

2) MIMO arrays: Contrarily to timed and phased arrays which perform accurate beamforming, MIMO arrays take 
advantage of the diversity gain ${ }^{3}$ provided by multiple orthogonal waveforms [28], [29] (see Fig. 2-(c)). To make the $\mathrm{Rx}$ able to discriminate the signal components coming from each single transmitting antenna, orthogonal waveforms are typically adopted [29], [30]. As an example, in [29] a class of signals (i.e., frequency spread signals) are demonstrated to maintain orthogonality for time delays and frequency Doppler shifts. This comes at the expense of a large bandwidth or long symbol duration time and of a higher complexity. In MIMO arrays, the normalized baseband transmitted signals are indicated with $P_{i}(f)=\mathcal{F}\left\{p_{i}(t)\right\}=\frac{1}{\sqrt{E}} \mathcal{F}\left\{s_{i}(t)\right\}$, where $\int\left|p_{i}(t)\right|^{2} d t=1, i=1,2, \ldots, N_{\mathrm{tx}}$. We consider orthogonal waveforms, such that the correlation function

$$
R_{p}\left(\Delta \tau_{i j}^{(l, k)}\right)=\int_{W} P_{i}(f) P_{j}^{*}(f) e^{-j 2 \pi f \Delta \tau_{i j}^{(l, k)}} d f
$$

is 0 when $i \neq j$ and $\neq 0$ otherwise, $\forall l, k=1, \ldots, L$, and where $\Delta \tau_{i j}^{(l, k)}=\tau_{i m l}-\tau_{j m k}$ with $m=1, \ldots, N_{\mathrm{rx}}$ and $i, j=1, \ldots, N_{\mathrm{tx}}$. The possibility to provide orthogonal waveforms permits to increase the diversity gain, as it will be detailed in the next sections, but it requires a greater bandwidth demand and a more complex Tx structure. In MIMO, the matrix in (5) is an identity matrix $\mathbf{B}\left(f, \boldsymbol{\theta}_{0}\right)=\mathbf{B}=\mathbf{I}_{N_{\mathrm{tx}}}$ and, in the presence of synchronization error, (6) becomes $\mathbf{Q}(f)=e^{-j 2 \pi\left(f+f_{\mathrm{c}}\right) \epsilon^{\mathrm{s}}} \mathbf{I}_{N_{\mathrm{tx}}}$.

3) Random Weighting: To avoid the complexity of MIMO, we propose a strategy relying on the same structure of phased arrays, i.e., with only PSs at each antenna branch (see Fig. 2(d)), with the fundamental difference that the value assigned to each PS is randomly chosen but known at the receiver. The beamforming matrix in (5) becomes

$$
\mathbf{B}\left(f, \boldsymbol{\theta}_{0}\right)=\mathbf{B}=\operatorname{diag}\left(e^{j v_{1}}, e^{j v_{2}}, \ldots, e^{j v_{i}}, \ldots, e^{j v_{N_{\mathrm{tx}}}}\right)
$$

with $v_{i} \sim \mathcal{U}(0,2 \pi)$. Note that in this configuration the matrix in (8) does not depend on the frequency and on the steering direction, thus resulting in an array pattern with a random shape [19], [23]. In the simplest implementation, random weighting could be realized using switches as discrete PSs randomly changing their status [20]. An important aspect is that, for both MIMO and random weighting, the rank of $\mathbf{B}$ is maximum and equal to $N_{\mathrm{tx}}$.

\section{B. Received Signal Model}

In this section, a general framework for the received signal model is illustrated. The received signals are collected in a vector $\mathbf{r}(f)=\left[R_{1}(f), \ldots, R_{m}(f), \ldots, R_{N_{\mathrm{rx}}}(f)\right]^{\mathrm{T}}$, where $R_{m}(f)=\mathcal{F}\left\{r_{m}(t)\right\}$ is evaluated in $T_{\text {obs }}$ and $r_{m}(t)$ is the received signal at the $m$ th receiving antenna. Specifically, the received signal vector can be written as

$$
\begin{aligned}
\mathbf{r}(f)=\sum_{l=1}^{L} & \mathbf{a}^{\mathrm{r}}\left(f, \boldsymbol{\theta}_{l}^{\mathrm{r}}, \boldsymbol{\vartheta}^{\mathrm{r}}\right) \mathbf{c}\left(f, \tau_{l}\right) \mathbf{A}^{\mathrm{t}}\left(f, \boldsymbol{\theta}_{l}^{\mathrm{t}}, \boldsymbol{\vartheta}^{\mathrm{t}}\right) \\
& \times \mathbf{Q}(f) \mathbf{B}\left(f, \boldsymbol{\theta}_{0}\right) \mathbf{s}(f)+\mathbf{n}(f)=\mathbf{x}(f)+\mathbf{n}(f)
\end{aligned}
$$

\footnotetext{
${ }^{3}$ Differently from communications, here we denote with "diversity gain" the possibility at the Rx side to distinguish among the transmitted signals (for example, in frequency domain) and, thus, to collect $N_{\mathrm{tx}} \times N_{\mathrm{rx}}$ independent measurements, thanks to the orthogonality between the waveforms.
}

where the Rx and Tx direction matrices are given by

$$
\begin{aligned}
& \mathbf{a}^{\mathrm{r}}\left(f, \boldsymbol{\theta}_{l}^{\mathrm{r}}\right)=\left[e^{j \gamma_{1}^{\mathrm{r}}}, \ldots, e^{j \gamma_{N_{\mathrm{rx}}}^{\mathrm{r}}}\right]^{\mathrm{T}} \\
& \mathbf{A}^{\mathrm{t}}\left(f, \boldsymbol{\theta}_{l}^{\mathrm{t}}, \boldsymbol{\vartheta}^{\mathrm{t}}\right)=\operatorname{diag}\left(e^{-j \gamma_{1}^{\mathrm{t}}}, \ldots, e^{-j \gamma_{N_{\mathrm{tx}}}^{\mathrm{t}}}\right)
\end{aligned}
$$

with $\gamma_{n}=2 \pi\left(f+f_{\mathrm{c}}\right) \tau_{n}\left(\boldsymbol{\theta}_{l}, \boldsymbol{\vartheta}\right)$. $\mathbf{c}\left(f, \tau_{l}\right)=c_{l} \mathbf{1}_{1 \times N_{\mathrm{tx}}}$ is the $1 \times N_{\mathrm{tx}}$ channel vector whose generic element is $c_{l}=$ $a_{l} e^{-j 2 \pi\left(f+f_{\mathrm{c}}\right) \tau_{l}}=\alpha_{l} e^{-j 2 \pi f \tau_{l}}$. Specifically, the dominant LOS component related to direct path (i.e., $l=1$ ) is considered deterministic while, for $l>1, \alpha_{l} \sim \mathcal{C N}\left(0, \sigma_{l}^{2}\right)$ is a circularly symmetric Gaussian random variable (RV) statistically modelling the $l$ th MPC [31].

Finally, $\quad \mathbf{x}(f)=\left[X_{1}(f), \ldots, X_{m}(f), \ldots, X_{N_{\mathrm{rx}}}(f)\right]^{\mathrm{T}}$ is the set of useful received signals and $\mathbf{n}(f)=$ $\left[N_{1}(f), \ldots, N_{m}(f), \ldots, N_{N_{\text {rx }}}(f)\right]^{\mathrm{T}}$ is the noise vector with $N_{m}(f)=\mathcal{F}\left\{n_{m}(t)\right\}$ and $n_{m}(t) \sim \mathcal{C N}\left(0, \sigma_{n}^{2}\right)$ with $\sigma_{n}^{2}=N_{0} W$ being $N_{0}$ the single-side noise power spectral density (PSD). For further convenience, define $\nu_{t}=E_{\mathrm{tot}} / N_{0}=\nu N_{\mathrm{tx}}$, with $\nu=E / N_{0}$. The SNR of the useful component (direct path) at each receiving antenna element is $\mathrm{SNR}=N_{\mathrm{tx}} \mathrm{SNR}_{1}$, where $\mathrm{SNR}_{1}=\left(a_{1}\right)^{2} \nu$ represents the SNR component related to the direct path between a generic couple of Tx-Rx antenna elements.

\section{Position ANd ORIEnTATION ERror Bound}

The aim of the estimation is to infer the position $\mathbf{p}^{t}$ of the Tx (e.g., mobile) and its orientation $\vartheta^{t}$ at the Rx side (e.g., the AP) starting from the set of received waveforms $\mathbf{r}(f)$.

The unknown parameters vector is defined as

$$
\boldsymbol{\psi}=\left[\mathbf{q}^{\mathrm{T}}, \boldsymbol{\kappa}^{\mathrm{T}}, \epsilon^{\mathrm{s}}\right]^{\mathrm{T}}=\left[\boldsymbol{\psi}_{\mathrm{nr}}, \boldsymbol{\psi}_{\mathrm{r}}\right]^{\mathrm{T}}
$$

where the parameters of interest are collected in

$$
\mathbf{q}=\left\{\begin{array}{ll}
{\left[\left(\mathbf{p}^{\mathrm{t}}\right)^{\mathrm{T}},\left(\boldsymbol{\vartheta}^{\mathrm{t}}\right)^{\mathrm{T}}\right]^{\mathrm{T}}} & \text { orientation-unaware } \\
\mathbf{p}^{\mathrm{t}} & \text { orientation-aware }
\end{array} .\right.
$$

As emerged from (12), we will discern among two different cases based on the knowledge or not of the orientation: the orientation-unaware case indicates the situation in which the Tx orientation is not known at $\mathrm{Rx}$ while the orientation-aware case is the opposite situation in which the orientation is exactly known at the Rx side and it can be removed from the unknown parameter vector. It will be clear from the results that only MIMO can deal with the orientation-unawareness situation; while beamforming fails in jointly estimating both parameters.

The multipath parameters are stacked in $\boldsymbol{\kappa}=\left[\boldsymbol{\kappa}_{1}^{\mathrm{T}}, \boldsymbol{\kappa}_{2}^{\mathrm{T}}, \ldots, \boldsymbol{\kappa}_{l}^{\mathrm{T}}, \ldots, \boldsymbol{\kappa}_{L}^{\mathrm{T}}\right]^{\mathrm{T}}$, with $\boldsymbol{\kappa}_{1}=\left[a_{1}\right]^{\mathrm{T}}$ and $\kappa_{l}=\left[\alpha_{l}^{\Re}, \alpha_{l}^{\Im}\right]^{\mathrm{T}}$ for $l>1$. The terms $\alpha_{l}^{\Re}=\Re\left\{\alpha_{l}\right\}$ and $\alpha_{l}^{\Im}=\Im\left\{\alpha_{l}\right\}$ indicate the real and imaginary part of the complex channel coefficient [22]. In this context, the MPCs and the residual synchronization error, i.e., $\boldsymbol{\psi}_{\mathrm{r}}=\left[\boldsymbol{\kappa}_{2}^{\mathrm{T}}, \ldots, \boldsymbol{\kappa}_{L}^{\mathrm{T}}, \epsilon^{\mathrm{s}}\right]^{\mathrm{T}}$, represent nuisance parameters when evaluating the ultimate performance of the estimator and, thus, a statistical a-priori information of these parameters is available. Differently, $\psi_{\mathrm{nr}}=\left[\mathbf{q}^{\mathrm{T}}, a_{1}\right]^{\mathrm{T}}$ are treated as deterministic (no a-priori information available).

In turns, this means that the multipath is not useful for retrieving the Tx position, as instead assumed in [5], [9] where 
the MPCs characteristics (i.e., delay, direction of arrival) bring useful location-related information. In fact, here we suppose that the multipath presents only random components that are uncorrelated with the Tx position. Consequently, if the LOS component is present together with MPCs, it is demonstrated in [15] that the CRB on the position is (negatively) affected only by the first-contiguous MPC cluster. Finally, we model the synchronization error as Gaussian zero-mean RV with standard deviation $\sigma_{\epsilon}^{2}$ [22], [32]-[34].

The performance of any unbiased estimator $\widehat{\psi}=\widehat{\psi}(\mathbf{r}(f))$ can be bounded by the CRB defined as [35]

$$
\mathbb{E}_{\mathbf{r}, \boldsymbol{\psi}_{\mathrm{r}}}\left\{[\widehat{\boldsymbol{\psi}}-\boldsymbol{\psi}][\widehat{\boldsymbol{\psi}}-\boldsymbol{\psi}]^{\mathrm{T}}\right\} \succeq \mathbf{J}_{\boldsymbol{\psi}}^{-1}=\operatorname{CRB}(\boldsymbol{\psi})
$$

where $\mathbf{J}_{\psi}$ is the Bayesian Fisher Information Matrix (FIM) defined as

$$
\begin{aligned}
& \mathbf{J}_{\boldsymbol{\psi}} \triangleq-\mathbb{E}_{\mathbf{r}, \boldsymbol{\psi}_{\mathrm{r}}}\left\{\nabla_{\boldsymbol{\psi} \boldsymbol{\psi}}^{2} \ln f\left(\mathbf{r}, \boldsymbol{\psi}_{\mathrm{r}}\right)\right\}=\mathbf{J}_{\boldsymbol{\psi}}^{\mathrm{d}}+\mathbf{J}_{\boldsymbol{\psi}}^{\mathrm{p}}
\end{aligned}
$$

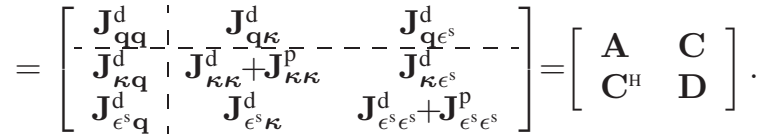

The symbol $\nabla_{\boldsymbol{\psi} \boldsymbol{\psi}}^{2}=\left(\partial^{2} / \partial \boldsymbol{\psi} \partial \boldsymbol{\psi}\right)$ denotes the second partial derivatives with respect to the elements in $\psi$ and

$$
\begin{aligned}
& \mathbf{J}_{\boldsymbol{\psi}}^{\mathrm{d}}=-\mathbb{E}_{\mathbf{r}, \boldsymbol{\psi}_{\mathrm{r}}}\left\{\nabla_{\boldsymbol{\psi} \boldsymbol{\psi}}^{2} \ln f\left(\mathbf{r} \mid \boldsymbol{\psi}_{\mathrm{r}}\right)\right\} \\
& \mathbf{J}_{\boldsymbol{\psi}}^{\mathrm{p}}=-\mathbb{E}_{\boldsymbol{\psi}_{\mathrm{r}}}\left\{\nabla_{\boldsymbol{\psi} \boldsymbol{\psi}}^{2} \ln f\left(\boldsymbol{\psi}_{r}\right)\right\}
\end{aligned}
$$

are the FIMs related to data $\left(\mathbf{J}_{\psi}^{\mathrm{d}}\right)$ and to the a-priori statistical information on the parameters $\left(\mathbf{J}_{\psi}^{\mathrm{p}}\right)$. Since the observations at each receiving antenna element are independent, the loglikelihood function $\left(\ln f\left(\mathbf{r} \mid \psi_{\mathrm{r}}\right)\right)$ can be written as

$$
\ln f\left(\mathbf{r} \mid \boldsymbol{\psi}_{\mathrm{r}}\right) \propto-\frac{1}{N_{0}} \sum_{m=1}^{N_{\mathrm{rx}}} \int_{W}\left|R_{m}(f)-X_{m}(f)\right|^{2} d f .
$$

Moreover, based on the statistical information of $\psi_{\mathrm{r}}$, it is possible to derive the a-priori probability density function of parameters $\psi_{\mathrm{r}}$ whose expression is reported in Appendix A. All FIMs are reported in details in Appendixes A and B.

Finally, by using the Schur complement, the CRB expression related to the localization and orientation estimation error can be easily derived as

$$
\operatorname{CRB}(\mathbf{q})=\left(\mathbf{A}-\mathbf{C} \mathbf{D}^{-1} \mathbf{C}^{\mathrm{H}}\right)^{-1} .
$$

Notably, (18) is a general bound valid for different setup (MIMO, timed, phased and random weighting arrays) and accounting for signal weights quantization effects, synchronization mismatch and multipath. Compact expressions can be derived from (18) for the specific geometric case described in Sec. II-A to get insights on the key parameters affecting the performance as will be done in Sec. VI.

Nevertheless, it is well known that the CRB is a meaningful metric only when the global ambiguities in (17) are negligible [35] and, hence, it must be carefully used when comparing different configurations. Such a condition is satisfied when operating at high SNR (asymptotic SNR regime) but, unfortunately, the required high SNRs cannot be in general obtained, especially at high frequencies. Therefore, in the following section, we demonstrate that the global ambiguities can be made negligible without imposing the SNR to be very large, but by letting the antenna array being massive (massive array regime). In particular, we aim to show that, under random $\mathrm{Rx}$ array orientations, the number of geometric configurations in which the ambiguities are not negligible vanishes as the number of receiving antennas increases.

\section{CRB TIGHTNESS IN MASSIVE ARRAY REGIME}

Traditionally used in radar systems [24], the AF is a powerful tool to investigate the presence of ambiguities and it can be derived from the maximum likelihood (ML) function discarding the thermal noise component. Let define the AF, normalized with respect to $N_{\text {tx }}$ and $N_{\text {rx }}$, as

$$
\mathrm{AF}(\mathbf{p}, \tilde{\mathbf{p}})=\left|\frac{T_{\mathrm{obs}}}{N_{\mathrm{tx}} N_{\mathrm{rx}}} \int_{W} \mathbf{x}^{\mathrm{H}}(f, \mathbf{p}) \mathbf{x}(f, \tilde{\mathbf{p}}) d f\right|^{2}
$$

where $\mathbf{p}$ is the true Tx position, $\tilde{\mathbf{p}}$ is the test position, $\mathbf{x}$ the useful signal vector reported in (9) and $T_{\text {obs }}$ indicates a suitable observation interval containing the signal support. Asymptotically for $N_{\mathrm{rx}} \rightarrow \infty$ (massive array regime) and considering different Rx orientations, for the weak law of the large numbers [36], we can write

$$
\mathrm{AF}(\mathbf{p}, \tilde{\mathbf{p}}) \stackrel{P}{\longrightarrow}\left|\frac{T_{\mathrm{obs}}}{N_{\mathrm{tx}} N_{\mathrm{rx}}} \int_{W} \mathbb{E}\left[\mathbf{x}^{\mathrm{H}}(f, \mathbf{p}) \mathbf{x}(f, \tilde{\mathbf{p}})\right] d f\right|^{2}
$$

where the operator $\stackrel{P}{\rightarrow}$ indicates the convergence in probability.

In the following, we will consider the free-space and the multipath cases, separately, in order to show how the sidelobes of the AF behave in the massive array regime. The analysis in non-massive array regime is considered in Sec. VIII.

\section{A. Free-space Scenario}

Here we focus our attention on the free-space scenario (i.e., $l=k=1$ ). In this case, the expectation term in (20) becomes

$$
\frac{1}{N_{\mathrm{tx}} N_{\mathrm{rx}}} \mathbb{E}\left[\mathbf{x}^{\mathrm{H}}(f, \mathbf{p}) \mathbf{x}(f, \tilde{\mathbf{p}})\right] \propto \frac{1}{N_{\mathrm{tx}} N_{\mathrm{rx}}} \mathbb{E}[\mathbf{H}(\mathbf{p}, \tilde{\mathbf{p}})]
$$

where $\mathbb{E}[\mathbf{H}(\mathbf{p}, \tilde{\mathbf{p}})]$ is a $N_{\mathrm{tx}} \times N_{\mathrm{tx}}$ matrix whose generic element is given by

$$
\begin{aligned}
& \mathbb{E}[\mathbf{H}(\mathbf{p}, \tilde{\mathbf{p}})]_{i, j}=\left|a_{1}\right|^{2} e^{-j 2 \pi\left(f+f_{\mathrm{c}}\right) \Delta \tau_{1}(\mathbf{p}, \tilde{\mathbf{p}})} \tilde{\omega}_{i} \tilde{\omega}_{j}^{*} e^{j \Psi_{i j}^{(1,1)}(\mathbf{p}, \tilde{\mathbf{p}})} \\
& \times \sum_{m=1}^{N_{\mathrm{rx}}} \mathbb{E}\left[e^{j \Psi_{m}^{(1,1)}\left(\mathbf{p}, \tilde{\mathbf{p}}, \boldsymbol{\vartheta}^{\mathrm{r}}\right)}\right]=\left\{\begin{array}{lr}
\left|a_{1}\right|^{2} N_{\mathrm{rx}} e^{j \Psi_{i j}^{(1,1)}(\mathbf{p}, \tilde{\mathbf{p}})} & \mathbf{p}=\tilde{\mathbf{p}} \\
0 & \text { otherwise }
\end{array}\right.
\end{aligned}
$$

with $\Delta \tau_{1}(\mathbf{p}, \tilde{\mathbf{p}})=\tau_{1}(\mathbf{p})-\tau_{1}(\tilde{\mathbf{p}}), \Psi_{i j}^{(1,1)}(\mathbf{p}, \tilde{\mathbf{p}})=\gamma_{i}^{\mathrm{t}}\left(\mathbf{p}, \boldsymbol{\vartheta}^{\mathrm{t}}\right)-$ $\gamma_{j}^{\mathrm{t}}\left(\tilde{\mathbf{p}}, \boldsymbol{\vartheta}^{\mathrm{t}}\right)$ and $\Psi_{m}^{(1,1)}\left(\mathbf{p}, \tilde{\mathbf{p}}, \boldsymbol{\vartheta}^{\mathrm{r}}\right)=-\gamma_{m}^{\mathrm{r}}\left(\mathbf{p}, \boldsymbol{\vartheta}^{\mathrm{r}}\right)+\gamma_{m}^{\mathrm{r}}\left(\tilde{\mathbf{p}}, \boldsymbol{\vartheta}^{\mathrm{r}}\right)$ which depends on the $\mathrm{Rx}$ array orientation. Note that $\Psi_{m}^{(1,1)}\left(\mathbf{p}, \tilde{\mathbf{p}}, \boldsymbol{\vartheta}^{\mathrm{r}}\right)=0$ for $\mathbf{p}=\tilde{\mathbf{p}}$ regardless the $\mathrm{Rx}$ orientation. On the other side, when $\mathbf{p} \neq \tilde{\mathbf{p}}$, in the presence of a large number of antenna elements $\left(N_{\mathrm{rx}} \rightarrow \infty\right)$ and considering random $\mathrm{Rx}$ orientations, the inter-antenna phase terms $\Psi_{m}\left(\mathbf{p}, \tilde{\mathbf{p}}, \boldsymbol{\vartheta}^{\mathrm{r}}\right)$ can be modeled as independent RVs uniformly distributed in $[0,2 \pi)$. In fact, different geometric 
configurations permit to span all the angles especially when large arrays are considered. ${ }^{4}$

This means that the percentage of geometrical configurations of the Rx for which the ambiguities are not negligible vanishes as $N_{\mathrm{rx}}$ increases (i.e., $\mathrm{AF}(\mathbf{p}, \tilde{\mathbf{p}}) \rightarrow 0$ when $\left.\mathbf{p} \neq \tilde{\mathbf{p}}\right)$. In other words, the conditions that permit to operate in the non-ambiguity region are twofold: the first is to increase the SNR (high-SNR regime) by keeping the number of antennas fixed, whereas the second fixes the SNR (even not extremely large) and lets the number of antennas grow.

\section{B. Multipath Scenario}

We now investigate if the CRB still remains a meaningful metric in the presence of multipath. To this end, we consider the AF by putting in evidence the multipath contribution as

$$
\begin{aligned}
& \mathrm{AF}(\mathbf{p}, \tilde{\mathbf{p}})=\left|\xi \int_{W} \mathbf{x}^{\mathrm{H}}(f, \mathbf{p}) \mathbf{x}(f, \tilde{\mathbf{p}}) d f\right|^{2} \\
& =\left|\xi \int_{W}\left(\mathbf{x}_{1}(f, \mathbf{p})+\mathbf{x}_{l>1}(f, \mathbf{p})\right)^{\mathrm{H}}\left(\mathbf{x}_{1}(f, \tilde{\mathbf{p}})+\mathbf{x}_{l>1}(f, \tilde{\mathbf{p}})\right) d f\right|^{2} \\
& =\left|\int_{W} \frac{f_{\mathrm{AWGN}}(\mathbf{p}, \tilde{\mathbf{p}})}{N_{\mathrm{tx}} N_{\mathrm{rx}}}+\frac{f_{\mathrm{MP}}(\mathbf{p}, \tilde{\mathbf{p}})}{N_{\mathrm{tx}} N_{\mathrm{rx}}} d f\right|^{2}
\end{aligned}
$$

where $\xi=\frac{T_{\text {obs }}}{N_{\text {ox }} N_{\text {rx }}}, \mathbf{x}_{1}(f, \mathbf{p})$ and $\mathbf{x}_{l>1}(f, \mathbf{p})$ indicate the expected received noise-free signal due to the direct path and multipath, respectively. Given the expression in (23), the following asymptotic analysis aims at verifying that the number of times the multipath impacts on the AF shape is negligible compared to the number of times it has not an effect at all, provided that the number of Rx antennas goes to infinity and that random array orientations are considered. More precisely, recalling the weak law of the large numbers, it is

$$
\frac{f_{\mathrm{MP}}(\mathbf{p}, \tilde{\mathbf{p}})}{N_{\mathrm{tx}} N_{\mathrm{rx}}} \stackrel{P}{\longrightarrow} \frac{1}{N_{\mathrm{tx}} N_{\mathrm{rx}}} \mathbb{E}\left[f_{\mathrm{MP}}(\mathbf{p}, \tilde{\mathbf{p}})\right]
$$

where we aim at testing if the right-hand side of (24) is 0 for $\mathbf{p} \neq \tilde{\mathbf{p}}$, meaning that AF sidelobes depending on multipath disappear when $N_{\mathrm{rx}}$ is large and random orientations are considered. The expectation argument in (24) is given by

$$
\begin{aligned}
& \mathbb{E}\left[f_{\mathrm{MP}}(\mathbf{p}, \tilde{\mathbf{p}})\right]=\mathbb{E}\left[\mathbf{x}_{l>1}^{\mathrm{H}}(f, \mathbf{p}) \mathbf{x}_{1}(f, \tilde{\mathbf{p}})\right] \\
& +\mathbb{E}\left[\mathbf{x}_{1}^{\mathrm{H}}(f, \mathbf{p}) \mathbf{x}_{l>1}(f, \tilde{\mathbf{p}})\right]+\mathbb{E}\left[\mathbf{x}_{l>1}^{\mathrm{H}}(f, \mathbf{p}) \mathbf{x}_{l>1}(f, \tilde{\mathbf{p}})\right] .
\end{aligned}
$$

Treating separately the terms in (25), we have

$$
\begin{aligned}
& \mathbb{E}\left[\mathbf{x}_{1}^{\mathrm{H}}(f, \mathbf{p}) \mathbf{x}_{l>1}(f, \tilde{\mathbf{p}})\right]=\sum_{m i j} \sum_{k=2}^{L} \alpha_{1}^{*} \alpha_{k} S_{i}(f) S_{j}^{*}(f) \tilde{\omega}_{i} \tilde{\omega}_{j}^{*} \\
& \times e^{-j 2 \pi f \tau_{k}} e^{-j \Psi_{i j}^{(1, k)}(\mathbf{p}, \tilde{\mathbf{p}})} \mathbb{E}\left[e^{j \Psi_{m}^{(1, k)}(\mathbf{p}, \tilde{\mathbf{p}})}\right]=0 \quad \forall \tilde{\mathbf{p}}
\end{aligned}
$$

where $\sum_{m i j}=\sum_{m=1}^{N_{\mathrm{rx}}} \sum_{i=1}^{N_{\mathrm{tx}}} \sum_{j=1}^{N_{\mathrm{tx}}}, \quad \Psi_{m}^{(1, k)}(\mathbf{p}, \tilde{\mathbf{p}})=$ $-\gamma_{m}\left(\boldsymbol{\theta}_{1}, \boldsymbol{\vartheta}^{\mathrm{r}}\right)+\gamma_{m}\left(\boldsymbol{\theta}_{k}, \boldsymbol{\vartheta}^{\mathrm{r}}\right), \Psi_{i j}^{(1, k)}=-\gamma_{i}\left(\boldsymbol{\theta}_{1}(\mathbf{p})\right)+\gamma_{j}\left(\boldsymbol{\theta}_{k}(\tilde{\mathbf{p}})\right)$, and $\mathbb{E}\left[e^{-j \Psi_{m}^{(1, k)}(\mathbf{p}, \tilde{\mathbf{p}})}\right]=0$ as the phases are assumed uniformly distributed between 0 and $2 \pi$. Similar considerations

\footnotetext{
${ }^{4}$ The goodness of the fitting with a uniform distribution has been validated through simulations.
}

are valid for $\mathbb{E}\left[\mathbf{x}_{l>1}^{\mathrm{H}}(f, \mathbf{p}) \mathbf{x}_{1}(f, \tilde{\mathbf{p}})\right]$. Finally, consider the last term in (25)

$$
\begin{aligned}
& \mathbb{E}\left[\mathbf{x}_{l>1}^{\mathrm{H}}(f, \mathbf{p}) \mathbf{x}_{l>1}(f, \tilde{\mathbf{p}})\right]=\sum_{m i j} \sum_{l=2}^{L} \sum_{k=2}^{L} S_{i}(f) S_{j}(f) \tilde{\omega}_{i} \tilde{\omega}_{j}^{*} \\
& \times e^{-j \Psi_{i j}^{(l, k)}(\mathbf{p}, \tilde{\mathbf{p}})} \alpha_{l} \alpha_{k}^{*} e^{-j 2 \pi f \Delta \tau_{l k}} \mathbb{E}\left[e^{-j \Psi_{m}^{(l, k)}(\mathbf{p}, \tilde{\mathbf{p}})}\right]
\end{aligned}
$$

where $\Delta \tau_{l k}=\tau_{l}-\tau_{k}, \Psi_{m}^{(l, k)}(\mathbf{p}, \tilde{\mathbf{p}})=\gamma_{m}^{\mathrm{r}}\left(\boldsymbol{\theta}_{l}, \mathbf{p}, \vartheta^{\mathrm{r}}\right)-$ $\gamma_{m}^{\mathrm{r}}\left(\boldsymbol{\theta}_{k}, \tilde{\mathbf{p}}, \boldsymbol{\vartheta}^{\mathrm{r}}\right), \Psi_{i j}^{(l, k)}(\mathbf{p}, \tilde{\mathbf{p}})=\gamma_{i}^{\mathrm{t}}\left(\boldsymbol{\theta}_{l}, \mathbf{p}\right)-\gamma_{i}^{\mathrm{t}}\left(\boldsymbol{\theta}_{k}, \tilde{\mathbf{p}}\right)$. In this case, since it holds

$$
\begin{aligned}
& \mathbb{E}\left[e^{-j \Psi_{m}^{(l, k)}(\mathbf{p}, \tilde{\mathbf{p}})}\right]= \\
& =\left\{\begin{array}{lll}
0 & \text { if } l \neq k, & \forall \tilde{\mathbf{p}} \\
1 & \text { if } l=k, & \mathbf{p}=\tilde{\mathbf{p}} \\
\mathbb{E}\left[e^{-j \Psi_{m}^{(l, l)}(\mathbf{p}, \tilde{\mathbf{p}})}\right]=0 & \text { if } l=k, & \mathbf{p} \neq \tilde{\mathbf{p}},
\end{array}\right.
\end{aligned}
$$

it follows that (27) is equal to 0 for $\mathbf{p} \neq \tilde{\mathbf{p}}$, i.e,. in all those cases where a global ambiguity can arise.

The obtained result shows that the global ambiguities due to the multipath are, on average, negligible. Nevertheless, the effect of multipath still remains in the correspondence of the true peak of the $\mathrm{AF}$, i.e., that for $\mathbf{p}=\tilde{\mathbf{p}}$, as reported in (28) for $l=k$. Consequently, even if we can state that the $\mathrm{CRB}$ is a valid metric in establishing the ultimate performance provided that $N_{\mathrm{rx}}$ is sufficiently large, the effect of multipath on the localization accuracy necessitates to be investigated. Before investigating such performance through simulations, next section will provide some insights on the performance limits in some specific scenarios.

\section{Free-Space Localization Bound}

This section provides an example on how the general CRB expression (18) can be simplified in absence of beamforming weights errors and MPCs. Specifically, in free-space conditions, (14) can be reduced to

$$
\mathbf{J}_{\psi}^{\mathrm{d}}=\mathbf{J}_{\psi}=\left[\begin{array}{cc}
\mathbf{J}_{\mathbf{q q}} & \mathbf{J}_{\mathbf{q} a_{1}} \\
\mathbf{J}_{a_{1} \mathbf{q}} & J_{a_{1} a_{1}}
\end{array}\right]=\left[\begin{array}{cc}
\mathbf{J}_{\mathbf{q q}}^{\mathrm{FS}} & \mathbf{0} \\
\mathbf{0} & J_{a_{1} a_{1}}
\end{array}\right]
$$

where its elements are reported in Appendix B and where the superscript ${ }^{d}$ is omitted as in this case all the parameters are deterministic. For readability convenience, we report here the expression of the FIM related to the localization parameters, that is

$$
\begin{aligned}
J_{q_{a} q_{b}}=8 \pi^{2} \nu a_{1}^{2} \sum_{m i j} \Re & \left\{\tilde{b}_{i j}^{\mathrm{c}} \xi_{i j}^{(1,1)} \chi_{i j}^{(1,1)}(2)\right\} \\
\times & \nabla_{q_{a}}\left(\tau_{i m 1}\right) \nabla_{q_{b}}\left(\tau_{j m 1}\right)
\end{aligned}
$$

where $q_{a}$ and $q_{b}$ represent two generic elements in the vector $\left\{x, y, z, \vartheta^{\mathrm{t}}, \varphi^{\mathrm{t}}\right\}$, and

$$
\chi_{i j}^{(1,1)}(2)=\int_{W} \tilde{b}_{i j}(f)\left(f+f_{\mathrm{c}}\right)^{2} e^{-j 2 \pi f \Delta \tau_{i j}^{(1,1)}} P_{i}(f) P_{j}^{*}(f) d f
$$

with $\Delta \tau_{i j}^{(1,1)}=\tau_{i m 1}-\tau_{j m 1}, \xi_{i j}^{(1,1)}=e^{-j 2 \pi f_{\mathrm{c}} \Delta \tau_{i j}^{(1,1)}}, \tilde{b}_{i j}(f)=$ $\tilde{b}_{i}(f) \tilde{b}_{j}^{*}(f)$, and $\tilde{b}_{i j}^{\mathrm{c}}=\tilde{b}_{i}^{\mathrm{c}}\left(\tilde{b}_{j}^{\mathrm{c}}\right)^{*}$. In (30), the derivatives translate the TOA and direction-of-arrival (DOA) in position 
and orientation information. In particular, for the position we have

$$
\nabla_{p}\left(\tau_{i m 1}\right)=\nabla_{p}\left(\tau_{1}\right)+\frac{\nabla_{p}\left(\boldsymbol{\theta}_{1}\right)}{c}\left[\mathbf{p}_{i}^{\mathrm{t}}\left(\boldsymbol{\vartheta}^{\mathrm{t}}\right)-\mathbf{p}_{m}^{\mathrm{r}}\left(\boldsymbol{\vartheta}^{\mathrm{r}}\right)\right]
$$

where $\nabla_{p}\left(\tau_{1}\right)$ highlights the dependence of the position from the direct path TOA and

$$
\nabla_{p}\left(\boldsymbol{\theta}_{1}\right)=\nabla_{p}\left(\theta_{1}\right) \nabla_{\theta_{1}}\left(\mathbf{d}\left(\boldsymbol{\theta}_{1}\right)\right)+\nabla_{p}\left(\phi_{1}\right) \nabla_{\phi_{1}}\left(\mathbf{d}\left(\boldsymbol{\theta}_{1}\right)\right)
$$

includes the dependence of the position from the DOA. Finally, for what the orientation information is regarded, we have

$$
\nabla_{\boldsymbol{\vartheta}^{\mathrm{t}}}\left(\tau_{i m 1}\right)=\nabla_{\boldsymbol{\vartheta}^{\mathrm{t}}}\left(\tau_{i}^{\mathrm{t}}\left(\boldsymbol{\theta}_{1}, \boldsymbol{\vartheta}^{\mathrm{t}}\right)\right)=\frac{1}{c} \mathbf{d}\left(\boldsymbol{\theta}_{1}\right) \nabla_{\boldsymbol{\vartheta}^{\mathrm{t}}}\left(\mathbf{p}_{i}^{\mathrm{t}}\left(\boldsymbol{\vartheta}^{\mathrm{t}}\right)\right) .
$$

By further analyzing (30), one can notice the dependence of the FIM from the beamforming weights given by the coefficients $\tilde{b}_{i j}^{\mathrm{c}}$ and $\tilde{b}_{i j}(f)$.

Starting from (29)-(30), it can be easily found that for beamforming and MIMO it is

$$
\mathrm{CRB}^{\mathrm{FS}}(\mathbf{q})=\left(\mathbf{J}_{\mathbf{q q}}^{\mathrm{FS}}\right)^{-1}=\left(\breve{\mathbf{J}}_{\mathbf{q q}}^{\mathrm{FS}} \mathbf{G}\right)^{-1}
$$

where we have separated the effect of signal design $\breve{\mathbf{J}}_{\mathbf{q q}}^{\mathrm{FS}}$, i.e., that related to (31), from that of the geometry $\mathbf{G}$, i.e., that related to (32)-(34). The matrix $\mathbf{G}$ provides, through derivatives, the relationship between the TOA at each Tx-Rx antenna element couple and the Tx position and orientation. In Appendix $\mathrm{C}$, this matrix is evaluated, considering the planar array geometry. Specifically for timed arrays, we have

$$
\breve{\mathbf{J}}_{\mathbf{q q}}^{\mathrm{FS}}=8 \pi^{2} \operatorname{SNR}_{1}\left(\beta^{2}+f_{\mathrm{c}}^{2}\right), \quad \mathbf{G}=\sum_{m i j} \nabla_{\mathbf{q q}}\left(\tau_{i m 1}, \tau_{j m 1}\right)
$$

where $\nabla_{\mathbf{q q}}\left(\tau_{i m 1}, \tau_{j m 1}\right)$ is a $5 \times 5$ matrix whose entries are given by $\nabla_{q_{a}}\left(\tau_{i m 1}\right) \nabla_{q_{b}}\left(\tau_{j m 1}\right)$, and $\beta$ is the baseband effective bandwidth of $p(t)$. Similarly, for MIMO arrays, it is possible to find

$$
\breve{\mathbf{J}}_{\mathbf{q q}}^{\mathrm{FS}}=8 \pi^{2} \mathrm{SNR}_{1}\left(\beta_{i}^{2}+f_{\mathrm{c}}^{2}\right), \mathbf{G}=\sum_{m i} \nabla_{\mathbf{q q}}\left(\tau_{i m 1}, \tau_{i m 1}\right)
$$

where $\sum_{m i}=\sum_{m=1}^{N_{\mathrm{rx}}} \sum_{i=1}^{N_{\mathrm{tx}}}$ and $\beta_{i}^{2}=\frac{\beta^{2}}{N_{\mathrm{tx}}}$ is the squared baseband effective bandwidth of $p_{i}(t)$.

\section{A. Special Case: Planar Arrays and Fixed Tx Orientation}

To improve the comprehension of (35)-(37) and to provide some insights on how the number of transmitting and receiving antennas can impact the performance, two particular cases of planar MIMO and timed arrays are discussed..$^{5}$ The planar array configuration has been described in Sec. II-A. More specifically, here we consider both arrays lying on the $X Z$-plane and being located one in front of the other with $\mathbf{p}^{\mathrm{r}}=[0,0,0]^{\mathrm{T}}$ and $\mathbf{p}^{\mathrm{t}}=[0, y, 0]^{\mathrm{T}}$, with $y>0$, so that $d=y$ and, consequently, $\tau_{1}=y / c$. Thus, we account for a fixed Tx and Rx orientation, i.e., $\boldsymbol{\vartheta}^{\mathrm{t}}=\boldsymbol{\vartheta}^{\mathrm{r}}=[0,0]^{\mathrm{T}}$. Note that the overall CRB analysis is still valid for any orientation.

\footnotetext{
${ }^{5}$ When using the planar 2D array, two possible specular solutions exist in the 3D space. Such ambiguity cannot be solved by simply starting from oneshot single antenna measurements. To that purpose, several possible methods have been already proposed to discriminate between ambiguous solutions, spanning from data fusion to Bayesian filtering [11], [26], [37], [38].
}

Moreover, we assume for now a free-space propagation condition so that $\boldsymbol{\theta}_{1}^{\mathrm{t}}=\boldsymbol{\theta}_{1}^{\mathrm{r}}=\boldsymbol{\theta}_{1}=\left[\theta_{1}, \phi_{1}\right]^{\mathrm{T}}=\left[\frac{\pi}{2},-\frac{\pi}{2}\right]^{\mathrm{T}}$ and $\mathbf{d}\left(\boldsymbol{\theta}_{1}\right)=[0,-1,0]$. Consequently, it is possible to formulate the inter-antenna propagation delay as

$$
\tau_{n}\left(\boldsymbol{\theta}_{1}, \boldsymbol{\vartheta}\right)=-\frac{d_{\mathrm{ant}}}{c}\left(n_{x} \sin (\varphi)+n_{z} \cos (\varphi) \sin (\vartheta)\right)
$$

where $n=1, \ldots, N$ is the antenna index while $n_{x}=n_{z}=$ $-\frac{\sqrt{N}-1}{2}, \ldots, \frac{\sqrt{N}-1}{2}$ are the indexes along the $x-$ and $z$-axis, respectively, and $N$ indicates the number of antennas (either at the Tx and Rx). Note that, when it is $\boldsymbol{\vartheta}^{\mathrm{r}}=\boldsymbol{\vartheta}^{\mathrm{t}}=[0,0]^{\mathrm{T}}$, the inter-antenna delays are zeros, i.e. $\tau_{m}^{\mathrm{r}}\left(\boldsymbol{\theta}_{1}, \boldsymbol{\vartheta}^{\mathrm{r}}\right)=\tau_{i}^{\mathrm{t}}\left(\boldsymbol{\theta}_{1}, \boldsymbol{\vartheta}^{\mathrm{t}}\right)=$ $0 \forall m, i$, as the antennas are aligned to the array centroids, thus the incident wave impinges simultaneously at all the antennas.

1) Planar MIMO Array: For the planar geometric configuration and in the orientation-unaware case, the diagonal elements in the position and orientation CRB matrix derived starting from (35)-(37), are given by

$$
\begin{aligned}
& \operatorname{CRB}(x)=\operatorname{CRB}(z)=\operatorname{CRB}_{0} \frac{12}{S\left(N_{\mathrm{rx}}-1\right)} \\
& \operatorname{CRB}(y)=\frac{\mathrm{CRB}_{0}}{N_{\mathrm{rx}}} \\
& \operatorname{CRB}\left(\vartheta^{\mathrm{t}}\right)=\operatorname{CRB}\left(\varphi^{\mathrm{t}}\right)=\operatorname{CRB}_{0} \frac{12\left(N_{\mathrm{tx}}+N_{\mathrm{rx}}-2\right)}{A_{\mathrm{rx}}\left(N_{\mathrm{tx}}-1\right)\left(N_{\mathrm{rx}}-1\right)}
\end{aligned}
$$

where $\mathrm{CRB}_{0}=c^{2} /\left(8 \pi^{2} \operatorname{SNR}\left(\beta_{i}^{2}+f_{\mathrm{c}}^{2}\right)\right)$ is the CRB of the ranging error one would obtain using only one antenna radiating an energy equal to $E_{\mathrm{tot}}=N_{\mathrm{tx}} E$, and $S=A^{\mathrm{r}} / y^{2}$ represents the ratio between the $\mathrm{Rx}$ array area and the squared $\mathrm{Tx}-\mathrm{Rx}$ distance. Note that $\mathrm{CRB}_{0}$ depends on the carrier frequency $f_{\mathrm{c}}{ }^{6}{ }^{6}$ on the shape of the pulse through $\beta_{i}^{2}$, on the received SNR, and it does not depend on the number of transmitting antennas. The analytical derivation is reported in Appendix C. From (39), it is possible to remark that the CRB of the estimation error in the $y$-coordinate is inversely proportional to the number of the receiving antenna elements accounting for the number of independent measurements available at the Rx. Regarding the other two coordinates, a key parameter on the estimation accuracy is $S$ which is related to the ratio between the dimension of the Rx array and the distance between the arrays: as this ratio becomes smaller (i.e., as the distance between the arrays becomes larger with respect to the array size), the positioning accuracy degrades. From (39) it is also possible to notice that the accuracy in estimating the orientation depends both on the transmitting and receiving antennas. Specifically both $N_{\text {tx }}$ and $N_{\text {rx }}$ must be greater than 1 to make the orientation possible whereas, for the positioning, only the number of receiving elements must be $>1$. Moreover, non-zero off-

\footnotetext{
${ }^{6}$ As we consider a coherent processing, the $\mathrm{Rx}$ is capable of retrieving the carrier phase of the incoming signal, evidenced by observing the presence of $f_{\mathrm{c}}$ in the expression of $\mathrm{CRB}_{0}$. The non-coherent case can be obtained by simply setting $f_{\mathrm{c}}=0$ and by taking into account only the baseband effective bandwidth. Non-perfect coherent processing can be taken into account through the synchronization error $\epsilon^{\mathrm{s}}$.
} 
diagonal elements remark a correlation between the error on the estimation of position and orientation. Specifically we have

$$
\begin{aligned}
\operatorname{CRB}\left(z, \vartheta^{\mathrm{t}}\right) & =\operatorname{CRB}\left(\vartheta^{\mathrm{t}}, z\right)=\operatorname{CRB}\left(x, \varphi^{\mathrm{t}}\right) \\
& =\operatorname{CRB}\left(\varphi^{\mathrm{t}}, x\right)=\operatorname{CRB}_{0} \frac{12}{S y\left(1-N_{\mathrm{rx}}\right)} .
\end{aligned}
$$

Contrarily in the orientation aware case, it can be found

$$
\begin{aligned}
& \operatorname{CRB}(x)=\operatorname{CRB}(z)=\operatorname{CRB}_{0} \frac{12}{S\left(N_{\mathrm{tx}}+N_{\mathrm{rx}}-2\right)} \\
& \operatorname{CRB}(y)=\frac{\mathrm{CRB}_{0}}{N_{\mathrm{rx}}} .
\end{aligned}
$$

Note that when passing from the condition of orientationunawareness to that of orientation-awareness, the positioning accuracy increases thanks to the additional information provided. In fact, the CRB on the $x$ - and $z$ - coordinates now depends also on the number of transmitting antennas.

2) Planar Timed Array: Differently from MIMO, here in the orientation-unaware case, the equivalent FIM for position and orientation is singular, meaning that it is not possible to jointly localize and determine the orientation using beamforming strategies.

If the $\mathrm{Tx}$ orientation is a known parameter (orientation aware case) and, thus, it is discarded from the estimation parameters vector, the elements of the position CRB matrix result from (57)

$$
\begin{aligned}
& \operatorname{CRB}(x)=\operatorname{CRB}(z)=\mathrm{CRB}_{0} \frac{12}{S} \frac{1}{N_{\mathrm{tx}}\left(N_{\mathrm{rx}}-1\right)} \\
& \mathrm{CRB}(y)=\frac{\mathrm{CRB}_{0}}{N_{\mathrm{tx}} N_{\mathrm{rx}}} .
\end{aligned}
$$

From (42) it is possible to remark that the CRB of the estimation error in the $y$-coordinate is inversely proportional to $N_{\text {tx }}$ and $N_{\text {rx }}$. In fact, the $N_{\text {tx }}$ term accounts for the SNR enhancement due to the beamforming process while $N_{\mathrm{rx}}$ for the number of independent measurements available at the Rx (receiver diversity). Note that when $N_{\text {rx }}=1$, the localization along the $x$ and $z$ axes is not possible (only ranging in the $y$ direction), as for MIMO.

More details related to the derivation of (42) are reported in Appendix C.

\section{MULTIPATH EFFECT ON LOCALIZATION ACCURACY}

Once verified that the CRB is still a meaningful metric in presence of multipath (refer to Sec. V-B), we now investigate its impact on the localization performance for the considered scenario. In [15], it is demonstrated that only the information related to the first-contiguous cluster, i.e. the set of MPCs overlapped to the first path, is relevant from a localization perspective in the asymptotic SNR regime. Here we show that under the asymptotic massive antenna regime, all the MPCs can be made negligible, included those belonging to the firstcontiguous cluster. The FIM in presence of multipath can be written as follows

$$
\mathbf{J}_{\psi}=\left[\begin{array}{ll}
\mathbf{J}_{\mathbf{q q}} & \mathbf{J}_{\mathbf{q} \kappa} \\
\mathbf{J}_{\kappa \mathbf{q}} & \mathbf{J}_{\kappa \kappa}
\end{array}\right]
$$

where $\mathbf{J}_{\kappa \kappa}$ contains also the a-priori information on MPCs statistics reported in Appendix A. Consequently, the CRB for the multipath scenario can be formulated as

$$
\operatorname{CRB}(\mathbf{q})=\left(\mathbf{J}_{\mathbf{q q}}-\mathbf{J}_{\mathbf{q} \kappa} \mathbf{J}_{\kappa \kappa}^{-1} \mathbf{J}_{\kappa \mathbf{q}}\right)^{-1}
$$

where all multipath information is gathered in $\mathbf{J}_{\mathbf{q} \kappa} \mathbf{J}_{\kappa \kappa}^{-1} \mathbf{J}_{\kappa \mathbf{q}}$. Considering the average over different Rx orientations and for large values of $N_{\mathrm{rx}}$, it is possible to show that the number of configurations where the multipath impacts the localization performance compared to the number of configurations in which it does not influence the accuracy is negligible regardless the chosen array architecture. Considering (44), for the weak law of the large numbers (i.e., for $N_{\text {rx }} \rightarrow \infty$ ), it holds

$$
\frac{1}{N_{\mathrm{rx}} N_{\mathrm{tx}}} \mathbf{J}_{\mathrm{q} \kappa} \stackrel{P}{\longrightarrow} \frac{1}{N_{\mathrm{rx}} N_{\mathrm{tx}}} \mathbb{E}\left[\mathbf{J}_{\mathrm{q} \kappa}\right]
$$

where in the following we aim at demonstrating that the righthand side goes to zero: $\frac{1}{N_{\text {rx }} N_{\text {tx }}} \mathbb{E}\left[\mathbf{J}_{\mathrm{q} \kappa}\right]=0$.

In the presence of a large number of antenna elements and considering random $\mathrm{Rx}$ orientations, the inter-antenna phase terms can be modeled as RVs uniformly distributed in $[0,2 \pi)$. Under this assumption, we have

$$
\begin{aligned}
& \mathbb{E}\left[J_{q a_{1}}\right]=J_{q a_{1}}=0 \\
& \mathbb{E}\left[J_{q \alpha_{k}^{\Re}}\right]= \\
& -4 \pi a_{1} \nu \sum_{m i j} \Im\left\{\tilde{b}_{i j}^{\mathrm{c}} \mathbb{E}\left[\xi_{i j}^{(k, 1)} \chi_{i j}^{(k, 1)}(1)\right]\right\} \nabla_{q}\left(\tau_{j m 1}\right)=0 \\
& \mathbb{E}\left[J_{q \alpha_{k}^{\Im}}\right]= \\
& -4 \pi a_{1} \nu \sum_{m i j} \Re\left\{\tilde{b}_{i j}^{\mathrm{c}} \mathbb{E}\left[\xi_{i j}^{(k, 1)} \chi_{i j}^{(k, 1)}(1)\right]\right\} \nabla_{q}\left(\tau_{j m 1}\right)=0
\end{aligned}
$$

where $\mathbb{E}\left[\xi_{i j}^{(k, 1)} \chi_{i j}^{(k, 1)}(1)\right] \propto \mathbb{E}\left[e^{-j 2 \pi\left(f+f_{\mathrm{c}}\right)\left(\Delta \tau_{m}^{\mathrm{r}}\left(\boldsymbol{\theta}_{1}, \boldsymbol{\theta}_{k}\right)\right)}\right]=0$ and with $\Delta \tau_{m}^{\mathrm{r}}\left(\boldsymbol{\theta}_{1}, \boldsymbol{\theta}_{k}\right)=\tau_{m}^{\mathrm{r}}\left(\boldsymbol{\theta}_{1}, \boldsymbol{\vartheta}^{\mathrm{r}}\right)-\tau_{m}^{\mathrm{r}}\left(\boldsymbol{\theta}_{k}, \boldsymbol{\vartheta}^{\mathrm{r}}\right)$. Similarly, it is straightforward to prove that the expectation of $\mathbf{J}_{\kappa \mathbf{q}}$ is zero.

The result in (45) leads to the important conclusion that letting the antennas array be massive, i.e., large $N_{\text {rx }}$, makes negligible the set of geometric configurations significantly impacted by MPCs, and the performance converges to that of the free-space case. As a consequence, the CRB converges to the $\mathrm{CRB}$ averaged over the $\mathrm{RX}$ orientations for massive antenna arrays.

\section{NUMERICAL RESULTS}

In this section, numerical results are reported considering different array configurations, multipath conditions and array non-idealities. Even though the signal model developed in this paper as well as the CRB derivation are valid for any frequency and bandwidth, here we focus our attention on mm-wave bands as an interesting case study for 5G/B5G scenarios thanks to the possibility to pack, in a small size, a larger number of antennas compared to what could be done at microwave bands.

For what the spatial deployment of the antennas is regarded, planar arrays are considered as they represent the most conventional structure to be integrated in APs and mobiles [13]. 
We recall that with fixed orientation we indicate the array configuration with the $\mathrm{Tx}$ and the Rx parallel to each other (i.e., the same described in Secs. II-A and VI-A), while with averaged orientation the geometric configuration in which, for each Monte Carlo iteration, a different 3D Rx array orientation is generated. If not otherwise indicated, the performance is shown for the orientation-aware case. In addition, we indicate with $\mathrm{Q}$ the presence of quantization errors, with $\mathrm{S}$ the presence of a residual synchronization error and with $\mathrm{O}$ the orientationunaware case.

Before analyzing MIMO and beamforming localization performance, it is necessary to ensure that the comparison based on $\mathrm{CRB}$ can be considered fair in terms of SNR working regimes when operating with non-massive arrays. To easy the reading, this analysis is reported in Appendix D.

\section{A. System Configuration}

We consider a scenario with a single AP equipped with a massive array, with the centroid placed in $\mathbf{p}^{\mathrm{r}}=[0,0,0]^{\mathrm{T}}$, and a transmitting array antenna whose centroid is located in $\mathbf{p}^{\mathrm{t}}=[0,5,0]^{\mathrm{T}}(d=5 \mathrm{~m})$. As in the mathematical model, the Rx has a perfect knowledge of the Tx steering direction, and the results are obtained for $f_{\mathrm{c}}=60 \mathrm{GHz}$ and $W=1 \mathrm{GHz}$ in free-space and multipath conditions. Root raised cosine (RRC) transmitted pulses centered at frequency $f_{\mathrm{c}}=60 \mathrm{GHz}$ and roll-off factor of 0.6 are adopted. A receiver noise figure of $N_{\mathrm{F}}=4 \mathrm{~dB}$ and a fixed transmitted power of $P_{\mathrm{t}}=10 \mathrm{~mW}$ are considered [39].

The performance is evaluated in terms of Position Error Bound (PEB) and Orientation Error Bound (OEB) defined as

$$
\mathrm{PEB}=\sqrt{\operatorname{tr}\left(\mathrm{CRB}\left(\mathbf{p}^{\mathrm{t}}\right)\right)}, \quad \mathrm{OEB}=\sqrt{\operatorname{tr}\left(\mathrm{CRB}\left(\boldsymbol{\vartheta}^{\mathrm{t}}\right)\right)}
$$

and averaged over $N_{\text {cycle }}=500$ Monte Carlo iterations.

For each cycle, a different 3D Rx array orientation, i.e., $\vartheta^{\mathrm{r}}=\left[\vartheta^{\mathrm{r}}, \varphi^{\mathrm{r}}\right]^{\mathrm{T}}$, and multipath scenario are generated. Specifically, when operating at $\mathrm{mm}$-wave frequencies, the paths timeof-arrival distributions can be described by a Poisson process and the inter-arrival times by an exponential probability density function [21], [40]. The arrival rate of paths is set to 4 [1/ns] while the azimuth and elevation AOA are modeled as uniformly distributed between $(0,2 \pi]$ and $(0, \pi]$, respectively. The array antennas are spaced apart of $d_{\text {ant }}=\lambda_{L} / 2$, where $\lambda_{L}=c / f_{L}$ and $f_{L}=f_{\mathrm{c}}-W / 2$. When present, the quantization errors of PSs are $\delta_{i}^{\mathrm{t}} \sim \mathcal{U}(-\pi / 4, \pi / 4)$ while the errors on TDLs are $\Delta \tau_{i}^{\mathrm{t}} \sim \mathcal{U}\left(0, d_{\text {ant }} / c\right)$. The standard deviation of the synchronization error is set to $\sigma_{\epsilon}=1 \mathrm{~ns}$.

\section{B. Results}

The results have been obtained as a function of: $N_{\mathrm{tx}}$ and $N_{\mathrm{rx}}$; the array configuration (i.e. timed, phased, random, MIMO); the presence and absence of array beamforming; the residual synchronization error; and the multipath overlapping effect.

1) Results in free-space: a) Impact of $N_{t x}$ : Figure 3 reports the PEB performance as a function of $N_{\mathrm{tx}}$ and of the $\mathrm{Rx}$ orientation in freespace. MIMO, timed and phased arrays are compared in the orientation-aware case when the number of receiving antennas is kept fixed to $N_{\text {rx }}=25$.

It can be observed that MIMO outperforms timed and phased arrays on average since it relies on different transmitted waveforms. On the contrary, as described in Sec. VI, arrays operating beamforming exhibit a better performance when the orientation is kept fixed. This is due to the fact that beamforming strategies fail in preserving the same accuracy for any geometric configuration (i.e., for any orientation). Contrarily, thanks to the diversity gain characterizing MIMO arrays, $\mathrm{Rx}$ orientations have a less significant effect on positioning accuracy.

For what the beamforming arrays are concerned, it can be observed that, as expected, timed and phased arrays have the same performance for $W / f_{\mathrm{c}} \ll 1$. Notably, phased arrays are the best candidate for narrowband systems where beamsteering operation can be accurately performed without the need to compensate for time delays. The impact of the fractional bandwidth on the timed/phased array performance has been investigated in [23]. Another important outcome from Fig. 3 is that array quantization errors, once characterized, slightly affect the localization performance. This implies that we can rely on simple array structures without severely affecting the performance. Finally, with $N_{\mathrm{tx}} \geq 25$, the performance improvement becomes less important if a sufficiently high $N_{\text {rx }}$ is considered. This implies that $N_{\text {tx }}$ can be relaxed to shrink the array dimensions and to enable the integration on portable devices [13]. Consequently, in the following, the number of transmitting antennas will be fixed to $N_{\mathrm{tx}}=25$.

b) Impact of $N_{r x}$ : In Fig. 4, the PEB performance is reported for both orientation-aware and-unaware cases as a function of $N_{\text {rx }}$ in free-space propagation condition, $N_{\mathrm{tx}}=25$ and fixed Tx and Rx orientation $\boldsymbol{\vartheta}^{\mathrm{t}}=\boldsymbol{\vartheta}^{\mathrm{r}}=[0,0]^{\mathrm{T}}$. Results, obtained using (39), (41) and (42), reveal that arrays performing beamforming outperform MIMO for the particular steering and geometric configuration conditions chosen, as already observed in Fig. 3. We ascribe the effect to an increased SNR in the considered direction. Nevertheless, with single-beam beamforming, orientation estimation is not always possible and, thus, the FIM results to be singular.

Figure 5 shows the average PEB performance when the Rx orientation randomly changes at each Monte Carlo iteration. For this analysis, we consider also random weighting, quantization errors as well as synchronization mismatch between the Tx and the Rx. As in Figs. 3-4, the performance of timed and phased arrays coincide due to the narrow fractional bandwidth (i.e., $W / f_{\mathrm{c}} \approx 0.016$ ). Differently from Fig. 4, in Fig. 5 MIMO achieves a higher positioning accuracy with respect to arrays employing beamforming strategies due to the fact that results are averaged over different $\mathrm{Rx}$ orientations. In fact, with MIMO, a reduction in the received SNR is experienced, but the number of independent measurements is maximized (i.e., $\left.N_{\text {tx }} N_{\text {rx }}\right)$.

For what random weighting arrays are regarded, they share the structure simplicity of phased arrays but they neither 


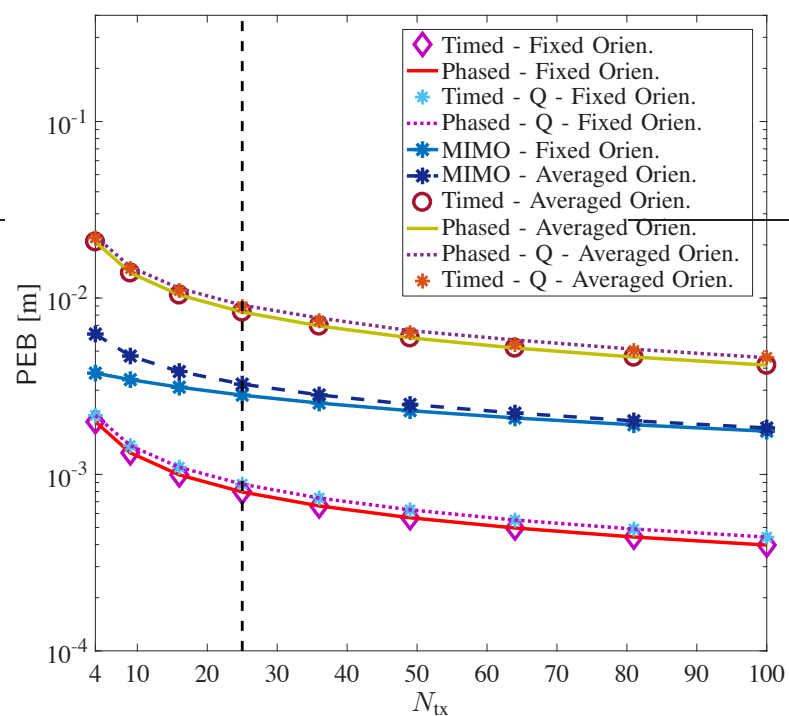

Fig. 3. PEB vs. $N_{\mathrm{tx}}, N_{\mathrm{rx}}=25$ and orientation aware.

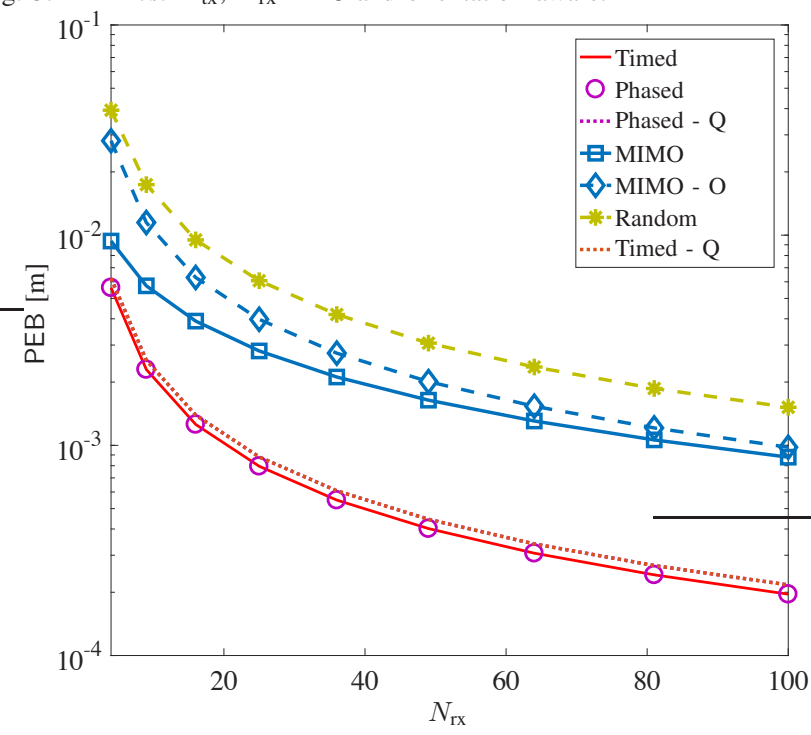

Fig. 4. PEB vs. $N_{\mathrm{rx}}, N_{\mathrm{tx}}=25$ and fixed Rx orientations.

perform beamforming nor achieve the diversity gain of MIMO, and thus, the positioning accuracy results degraded with respect to other structures. Nevertheless, if the requirement on the localization accuracy is not so stringent, they could be an interesting option to guarantee both a sub-centimeter positioning accuracy (e.g., for $N_{\mathrm{rx}}=50$ and $N_{\mathrm{tx}}=25, \mathrm{PEB} \approx 7 \mathrm{~mm}$ ) and an easy implementation in future devices operating at $\mathrm{mm}$-wave frequencies. Note that when the Tx orientation is one of the parameters to be estimated (orientation-unaware case), only MIMO presents a non-singular FIM. Obviously, in this case, given the reduced information available at the Rx side, the positioning accuracy worsen with respect to the orientation-aware case. In all configurations, the residual synchronization error significantly degrades the localization performance. In [41] solutions to avoid synchronization are proposed. Figure 6 reports the OEB as a function of $N_{\mathrm{rx}}$. In this case, only the performance of MIMO is present because of the singularity problem arising in timed, phased and random

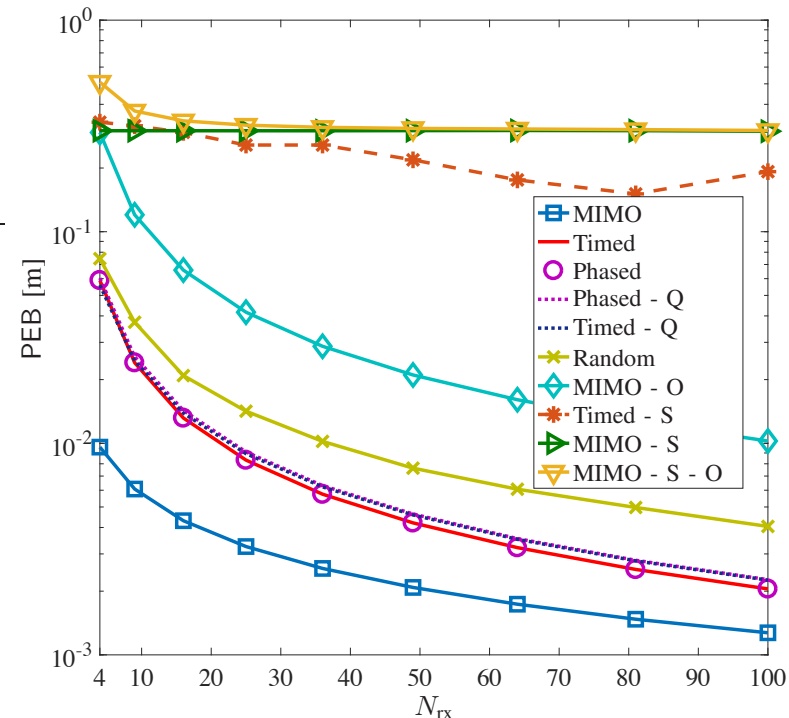

Fig. 5. PEB vs. $N_{\mathrm{rx}}, N_{\mathrm{tx}}=25$ and averaged Rx orientation.

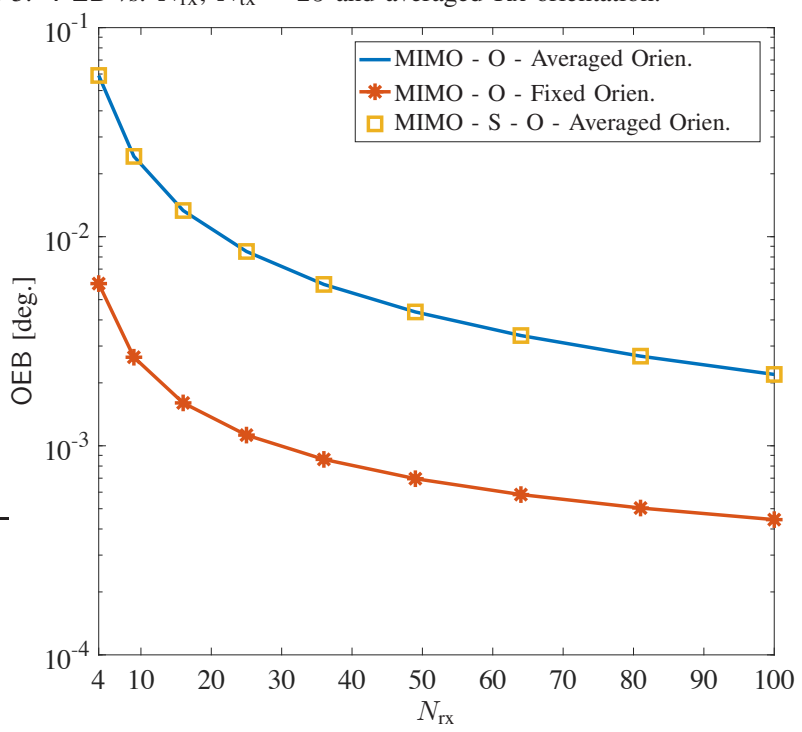

Fig. 6. OEB vs. $N_{\mathrm{rx}}, N_{\mathrm{tx}}=25$.

weighting arrays. An interesting result is that, in this case, the synchronization error does not impact the orientation accuracy.

c) Grid results: Figure 7 reports the PEB results for the orientation-aware case when the mobile moves in a grid of points spaced of $0.5 \mathrm{~m}$ considering phased (Fig. 7-top) and MIMO (Fig. 7-bottom) arrays, respectively. We considered a 3D indoor scenario of $10 \times 10 \times 3 \mathrm{~m}^{3}$ where the mobile and the AP centroids are at the same height. The $\mathrm{Rx}$, equipped with $N_{\mathrm{rx}}=100$ antennas, is placed in $\mathbf{p}^{\mathrm{r}}=[0,0,0]^{\mathrm{T}}$ with orientation changing at each Monte Carlo iteration. On the other side, the mobile array is equipped with $N_{\mathrm{tx}}=25$ elements and its orientation and its steering direction are fixed to $\vartheta^{\mathrm{t}}=[0,0]^{\mathrm{T}}$ and to the broadside direction, respectively. Grid results confirm that MIMO arrays localization performance does not depend on the Rx orientation and on mobile position in space but only on the distance between the Tx and the Rx. Indeed, from Fig. 7, it can be seen that if the steering angle is fixed, the localization accuracy is higher in a privileged direc- 

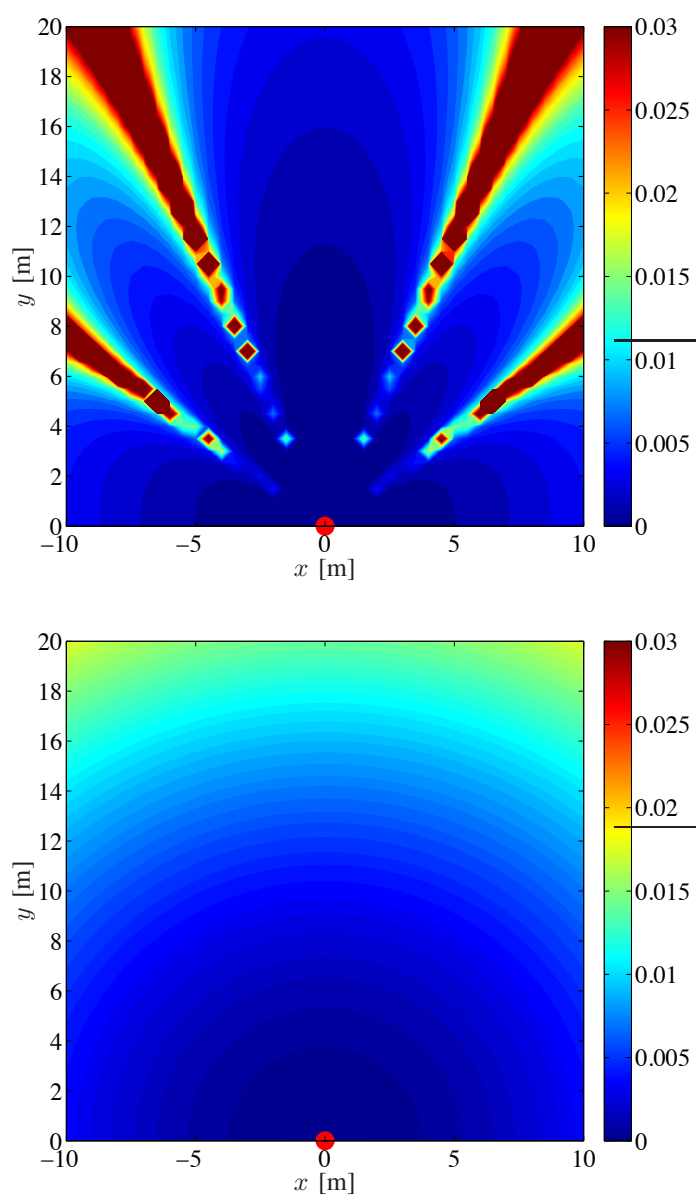

Fig. 7. Phased (top) and MIMO (bottom) array PEB [m], $N_{\mathrm{rx}}=100$, $N_{\mathrm{tx}}=25$ and averaged receiver orientations.

tion in space corresponding to the best geometric configuration conditions.

2) Multipath scenario: Figure 8 investigates the multipath effect by analysing the PEB and OEB averaged over different $\mathrm{Rx}$ orientations and as a function of the number of MPCs for phased and MIMO arrays. We consider the statistical multipath parameters described in Sec. IV. ${ }^{7}$ As foreseen in the asymptotic analysis in Sec. VII, when increasing $N_{\text {rx }}$, the MPCs effect becomes negligible and the performance tends to coincide with that obtained in free-space $(L=1)$. Moreover, it is possible to remark that phased arrays are more sensitive to multipath with respect to MIMO, especially when the number of receiving antennas is small. In fact, for phased arrays at least 25 antennas are necessary to make the MPCs negligible.

\section{CONCLUSION}

In this paper, we have considered an appealing scenario for $5 \mathrm{G} / \mathrm{B} 5 \mathrm{G}$ applications where a single-anchor localization exploiting massive arrays has been put forth. The theoretical localization performance has been evaluated by deriving the position and orientation CRB for different array configurations

\footnotetext{
${ }^{7}$ Note that the sparsity of the MPCs plays an additional role in the low impact of the multipath.
}
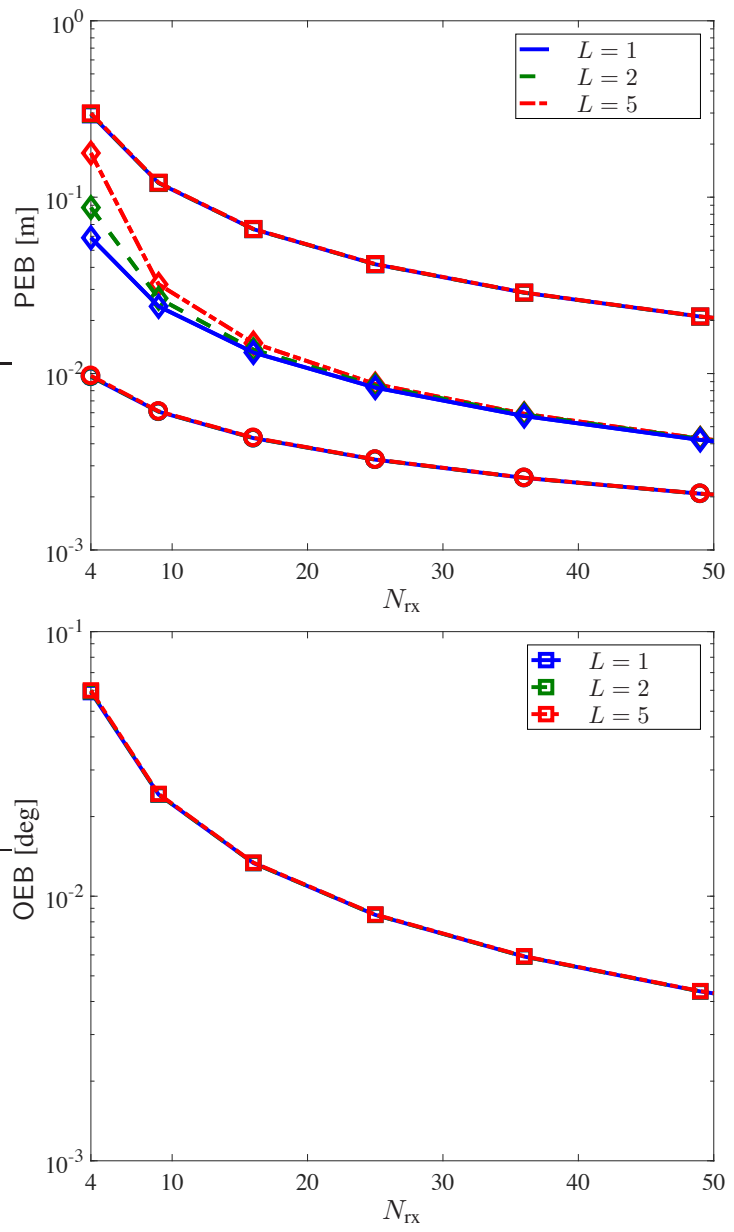

Fig. 8. PEB and OEB vs. $N_{\mathrm{rx}}$ in multipath propagation scenario, $N_{\mathrm{tx}}=25$, averaged receiver orientation. Diamond marked lines refer to phased array, circle marked lines to MIMO, and square marked lines to MIMO orientation unaware. Note that most plots are overlapped.

(i.e., MIMO vs. beamforming). Phase quantization, residual synchronization mismatch, and multipath have been considered as nuisance parameters in the estimation process.

From analytical and simulation results, the main achievements emerged in this paper can be summarized as follows:

- We show through an asymptotic analysis (i.e., massive array regime $N_{\mathrm{rx}} \rightarrow \infty$ ) that the considered CRB is a tight bound regardless the propagation condition and the array configuration.

- Thanks to the diversity gain exploitable for retrieving positioning information, MIMO outperforms beamforming in terms of localization and orientation performance when averaging over different geometric configurations. Nevertheless, the beamforming is desirable to maximize the SNR towards a specific direction in specific geometric configurations.

- Quantization errors slightly impact the localization performance and, thus, the array design requirements can be relaxed in favor of a lower complexity. The same considerations can be drawn when using the proposed random weighting method. Contrarily, a synchronization mismatch between the Tx and the Rx, affecting both 
the delay and phase retrieval, significantly degrades the positioning performance.

- The adoption of massive antenna arrays makes the positioning insensitive to multipath for most of geometric configurations regardless the SNR regime. We demonstrated such point through an asymptotic analysis valid for $N_{\text {rx }} \rightarrow \infty$. This is true even when the number of antennas is not extremely high (i.e., $N_{\mathrm{tx}}, N_{\mathrm{rx}}>20$ ) for relatively high SNR regime.

\section{APPENDIX A}

Considering the subset of random parameters $\psi_{\mathrm{r}}=$ $\left[\boldsymbol{\kappa}_{2}^{\mathrm{T}}, \ldots, \boldsymbol{\kappa}_{L}^{\mathrm{T}}, \epsilon^{\mathrm{s}}\right]^{\mathrm{T}}=\left[\boldsymbol{\alpha}^{\Re \mathrm{T}}, \boldsymbol{\alpha}^{\Im \mathrm{T}}, \epsilon^{\mathrm{s}}\right]^{\mathrm{T}}$, and treating them as independent RVs we can write $\ln \left(f\left(\boldsymbol{\psi}_{\mathrm{r}}\right)\right)=\ln \left(f\left(\boldsymbol{\alpha}^{\Re \mathrm{T}}\right)\right)+$ $\ln \left(f\left(\boldsymbol{\alpha}^{\Im \mathrm{T}}\right)\right)+\ln \left(f\left(\epsilon^{\mathrm{s}}\right)\right)$. Therefore, the elements of the $a$ priori FIM are

$$
J_{\epsilon^{\mathrm{s}} \epsilon^{\mathrm{s}}}^{\mathrm{p}}=\frac{1}{\sigma_{\epsilon}^{2}}, \quad J_{\alpha_{k}^{\Re} \alpha_{l}^{\Re}}^{\mathrm{p}}=J_{\alpha_{k}^{\Im} \alpha_{l}^{\Im}}^{\mathrm{p}}=\left\{\begin{array}{cc}
\frac{1}{\sigma_{l}^{2}} & \text { if } l=k \\
0 & \text { if } l \neq k .
\end{array}\right.
$$

\section{APPENDIX B}

In this Appendix we derive the elements of the data FIM reported in (15). To accomplish this task, we introduce the following quantities

$$
\begin{aligned}
& \chi_{i j}^{(l, k)}(p)=\int_{W} \tilde{b}_{i j}(f)\left(f+f_{\mathrm{c}}\right)^{p} e^{-j 2 \pi f \Delta \tau_{i j}^{(l, k)}} P_{i}(f) P_{j}^{*}(f) d f \\
& R_{i j}^{p}(\Delta \tau)=\int_{W} \tilde{b}_{i j}(f) e^{-j 2 \pi f \Delta \tau} P_{i}(f) P_{j}^{*}(f) d f \\
& R_{i j}^{\ddot{p}}(\Delta \tau)=\int_{W} \tilde{b}_{i j}(f) f^{2} e^{-j 2 \pi f \Delta \tau} P_{i}(f) P_{j}^{*}(f) d f
\end{aligned}
$$

where $\Delta \tau_{i j}^{(l, k)}=\tau_{i m l}-\tau_{j m k}, \tilde{b}_{i j}(f)=\tilde{b}_{i}(f) \tilde{b}_{j}^{*}(f)$, and $\tilde{b}_{i j}^{\mathrm{c}}=$ $\tilde{b}_{i}^{\mathrm{c}}\left(\tilde{b}_{j}^{\mathrm{c}}\right)^{*}$. The elements of $\mathbf{J}_{\mathbf{q q}}^{\mathrm{d}}$ can be expressed as in (30). The elements of $\mathbf{J}_{\kappa \kappa}^{\mathrm{d}}$ are

$$
\begin{aligned}
& J_{a_{1} a_{1}}=2 \nu \sum_{m i j} \Re\left\{\tilde{b}_{i j}^{\mathrm{c}} \xi_{i j}^{(1,1)} R_{i j}^{p}\left(\Delta \tau_{i j}^{(1,1)}\right)\right\} \\
& J_{\alpha_{k}^{\Re} a_{1}}=J_{a_{1} \alpha_{k}^{\Re}}^{\mathrm{H}}=2 \nu \sum_{m i j} \Re\left\{\tilde{b}_{i j}^{\mathrm{c}} \xi_{i j}^{(1, k)} R_{i j}^{p}\left(\Delta \tau_{i j}^{(1, k)}\right)\right\} \\
& J_{\alpha_{k}^{\Im} a_{1}}=J_{a_{1} \alpha_{k}^{\Im}}^{\mathrm{H}}=2 \nu \sum_{m i j} \Im\left\{\tilde{b}_{i j}^{\mathrm{c}} \xi_{i j}^{(1, k)} R_{i j}^{p}\left(\Delta \tau_{i j}^{(1, k)}\right)\right\} \\
& J_{\alpha_{k}^{\Re} \alpha_{l}^{\Re}}=J_{\alpha_{k}^{\Im} \alpha_{l}^{\Im}}=2 \nu \sum_{m i j} \Re\left\{\tilde{b}_{i j}^{\mathrm{c}} \xi_{i j}^{(l, k)} R_{i j}^{p}\left(\Delta \tau_{i j}^{(l, k)}\right)\right\} \\
& J_{\alpha_{k}^{\Im} \alpha_{l}^{\Re}}=J_{\alpha_{l}^{\Re} \alpha_{k}^{\Im}}^{\mathrm{H}}=2 \nu \sum_{m i j} \Im\left\{\tilde{b}_{i j}^{\mathrm{c}} \xi_{i j}^{(l, k)} R_{i j}^{p}\left(\Delta \tau_{i j}^{(l, k)}\right)\right\}
\end{aligned}
$$

where $\xi_{i j}^{(1, k)}=e^{-j 2 \pi f_{\mathrm{c}}\left(\tau_{i m 1}+\epsilon^{\mathrm{s}}+\tau_{m}^{\mathrm{r}}\left(\boldsymbol{\theta}_{k}\right)-\tau_{j}^{\mathrm{t}}\left(\boldsymbol{\theta}_{k}\right)\right)}$ and $\xi_{i j}^{(l, k)}=$ $e^{-j 2 \pi f_{\mathrm{c}}\left(-\tau_{m}^{\mathrm{r}}\left(\boldsymbol{\theta}_{l}\right)+\tau_{m}^{\mathrm{r}}\left(\boldsymbol{\theta}_{k}\right)+\tau_{i}^{\mathrm{t}}\left(\boldsymbol{\theta}_{l}\right)-\tau_{j}^{\mathrm{t}}\left(\boldsymbol{\theta}_{k}\right)\right)}$. The elements of $\mathbf{J}_{\mathbf{q} \boldsymbol{k}}^{\mathrm{d}}$ are

$$
\begin{aligned}
& J_{q a_{1}}=4 \pi a_{1} \nu \sum_{m i j} \Im\left\{\tilde{b}_{i j}^{\mathrm{c}} \xi_{i j}^{(1,1)} \chi_{i j}^{(1,1)}(1)\right\} \nabla_{q}\left(\tau_{j m 1}\right)=0 \\
& J_{q \alpha_{k}^{\Re}}=-4 \pi a_{1} \nu \sum_{m i j} \Im\left\{\tilde{b}_{i j}^{\mathrm{c}} \xi_{i j}^{(k, 1)} \chi_{i j}^{(k, 1)}(1)\right\} \nabla_{q}\left(\tau_{j m 1}\right)
\end{aligned}
$$

$$
J_{q \alpha_{k}^{\Im}}=-4 \pi a_{1} \nu \sum_{m i j} \Re\left\{\tilde{b}_{i j}^{\mathrm{c}} \xi_{i j}^{(k, 1)} \chi_{i j}^{(k, 1)}(1)\right\} \nabla_{q}\left(\tau_{j m 1}\right)
$$

where $\xi_{i j}^{(k, 1)}=e^{-j 2 \pi f_{\mathrm{c}}\left(-\tau_{j m 1}-\epsilon^{\mathrm{s}}-\tau_{m}^{\mathrm{r}}\left(\boldsymbol{\theta}_{k}\right)+\tau_{i}^{\mathrm{t}}\left(\boldsymbol{\theta}_{\boldsymbol{k}}\right)\right)}$. Now, if we consider the presence of a residual synchronization error, the FIM $\mathbf{J}_{\epsilon^{\mathrm{s}} \epsilon^{\mathrm{s}}}^{\mathrm{d}}$ is

$$
\begin{array}{r}
J_{\epsilon^{\mathrm{s}} \epsilon^{\mathrm{s}}}=8 \pi^{2} \nu \Re\left\{\sum _ { m i j } \tilde { b } _ { i j } ^ { \mathrm { c } } \left[a_{1}^{2} \xi_{i j}^{(1,1)} \chi_{i j}^{(1,1)}(2)+\right.\right. \\
\left.\left.\sum_{k=2}^{L} \sigma_{l}^{2} R_{i j}^{\ddot{p}}\left(\Delta \tau_{i j}^{(k, k)}\right) e^{\left.-j 2 \pi f_{\mathrm{c}}\left(\tau_{i}^{\mathrm{t}}\left(\boldsymbol{\theta}_{k}\right)-\tau_{j}^{\mathrm{t}}\left(\boldsymbol{\theta}_{k}\right)\right)\right]}\right]\right\} .
\end{array}
$$

Finally, the elements of $\mathbf{J}_{\boldsymbol{\kappa} \epsilon^{\mathrm{s}}}^{\mathrm{d}}$ and $\mathbf{J}_{\mathbf{q} \epsilon^{\mathrm{s}}}^{\mathrm{d}}$ are

$$
\begin{aligned}
& J_{a_{1} \epsilon^{\mathrm{s}}}=4 \pi a_{1} \nu \sum_{m i j} \Im\left\{\tilde{b}_{i j}^{\mathrm{c}} \xi_{i j}^{(1,1)} \chi_{i j}^{(1,1)}(1)\right\} \\
& J_{\alpha_{k}^{\Re} \epsilon^{\mathrm{s}}}=4 \pi a_{1} \nu \sum_{m i j} \Im\left\{\tilde{b}_{i j}^{\mathrm{c}} \xi_{i j}^{(1, k)} \chi_{i j}^{(1, k)}(1)\right\} \\
& J_{\alpha_{k}^{\Im} \epsilon^{\mathrm{s}}}=4 \pi a_{1} \nu \sum_{m i j} \Re\left\{\tilde{b}_{i j}^{\mathrm{c}} \xi_{i j}^{(1, k)} \chi_{i j}^{(1, k)}(1)\right\} \\
& J_{q \epsilon^{\mathrm{s}}}=8 \pi^{2} \nu a_{1}^{2} \sum_{m i j} \Re\left\{\tilde{b}_{i j}^{\mathrm{c}} \xi_{i j}^{(1,1)} \chi_{i j}^{(1,1)}(2)\right\} \nabla_{q}\left(\tau_{j m 1}\right) .
\end{aligned}
$$

\section{APPENDIX C}

In this Appendix we specialize the expression of the symmetric matrix $\mathbf{G}$ reported in (35)-(37). To this end, we explicit the geometric relationship relating the TOA between each Tx-Rx antennas couple and the considered localization parameter, i.e., $\nabla_{q_{a} q_{b}}\left(\tau_{i m l}, \tau_{j m l}\right)=\nabla_{q_{a}}\left(\tau_{i m l}\right) \nabla_{q_{b}}\left(\tau_{j m l}\right)$. For the particular antenna configuration chosen and described in Sec. II-A, in which the array antennas are spaced of $d_{\text {ant }}$, and considering $\boldsymbol{\vartheta}^{\mathrm{t}}=[0,0]^{\mathrm{T}}$, we can compute a simplified version of (32)-(34). Specifically, it is possible to obtain:

$$
\begin{aligned}
\nabla_{p}\left(\tau_{i m 1}\right) & =\frac{1}{c}\left[c \nabla_{p}\left(\tau_{1}\right)+d_{\mathrm{ant}}\left(\left(i_{x}-m_{x}\right) \nabla_{p}\left(\phi_{1}\right)\right.\right. \\
& \left.\left.+\left(m_{z}-i_{z}\right) \nabla_{p}\left(\theta_{1}\right)\right)\right] \\
\nabla_{\vartheta^{\mathrm{t}}}\left(\tau_{i m 1}\right) & =-\frac{d_{\mathrm{ant}}}{c} i_{z}, \quad \nabla_{\varphi^{\mathrm{t}}}\left(\tau_{i m 1}\right)=-\frac{d_{\mathrm{ant}}}{c} i_{x}
\end{aligned}
$$

with $m_{x}=m_{z}=-\frac{\sqrt{N_{\mathrm{rx}}}-1}{2},-\frac{\sqrt{N_{\mathrm{rx}}}-1}{2}+1, \ldots, \frac{\sqrt{N_{\mathrm{rx}}}-1}{2}$ and $i_{x}=i_{z}=j_{x}=j_{z}=-\frac{\sqrt{N_{\mathrm{tx}}}-1}{2},-\frac{\sqrt{N_{\mathrm{tx}}}-1}{2}+1, \ldots, \frac{\sqrt{N_{\mathrm{tx}}}-1}{2}$.

From (53)-(54), it is straightforward to derive (55). Then, by considering the summations present in $\mathbf{G}$, it is possible to obtain the CRB matrices for MIMO and timed arrays, respectively, as in (56)-(57) where $S=A^{\mathrm{r}} / y^{2}$.

\section{APPENDIX D}

In this Appendix we consider the AF as defined in (19). The AF for the position coordinates shows a main peak in correspondence of the true Tx position and secondary sidelobe peaks relative to "wrong" positions. An ambiguity problem arises when one of these sidelobes overcomes or becomes comparable to the main beam due to noise. Consequently, to determine whether ambiguities are negligible in the nonmassive array case, we have derived a threshold on the noise standard deviation to keep the ambiguity probability fixed to a desired low value. 


$$
\nabla_{\mathbf{q q}}\left(\tau_{i m 1}, \tau_{j m 1}\right)=\frac{d_{\text {ant }}^{2}}{(c y)^{2}}\left[\begin{array}{lllll}
\left(m_{x}-i_{x}\right)\left(m_{x}-j_{x}\right) & \frac{y}{d_{\text {ant }}}\left(m_{x}-i_{x}\right) & \left(m_{x}-i_{x}\right)\left(j_{z}-m_{z}\right) & y\left(i_{x}-m_{x}\right) j_{z} \ldots & y\left(i_{x}-m_{x}\right) j_{x} \\
\cdots & \frac{y^{2}}{d_{\text {ant }}^{2}} & \frac{y}{d_{\text {ant }}}\left(j_{z}-m_{z}\right) & -\frac{y^{2}}{d_{\text {ant }}} j_{z} & -\frac{y^{2}}{d_{\text {ant }}} j_{x} \\
\cdots & \cdots & \left(i_{z}-m_{z}\right)\left(j_{z}-m_{z}\right) & y\left(m_{z}-i_{z}\right) j_{z} & y\left(m_{z}-i_{z}\right) j_{x} \\
\cdots & \cdots & \cdots & y^{2} i_{z} j_{z} & y^{2} i_{z} j_{x} \\
\cdots & \cdots & \cdots & \cdots & y^{2} i_{x} j_{x}
\end{array}\right]
$$

$$
\begin{aligned}
& \operatorname{CRB}(\mathbf{q})=\frac{c^{2}}{8 \pi^{2} N_{\mathrm{tx}} \mathrm{SNR}_{1}\left(\beta_{i}^{2}+f_{\mathrm{C}}^{2}\right)} \frac{1}{S}\left[\begin{array}{lllll}
\frac{12}{\left(N_{\mathrm{rx}}-1\right)} & 0 & 0 & 0 & \frac{12}{y\left(1-N_{\mathrm{rx}}\right)} \\
0 & \frac{S}{N_{\mathrm{rx}}} & 0 & 0 & 0 \\
0 & 0 & \frac{12}{\left(N_{\mathrm{rx}}-1\right)} & \frac{12}{y\left(1-N_{\mathrm{rx}}\right)} & 0 \\
0 & 0 & \frac{12}{y\left(1-N_{\mathrm{rx}}\right)} & \frac{12\left(N_{\mathrm{rx}}+N_{\mathrm{tx}}-2\right)}{y^{2}\left(N_{\mathrm{tx}}-1\right)\left(N_{\mathrm{rx}}-1\right)} & 0 \\
\frac{12}{y\left(1-N_{\mathrm{rx}}\right)} & 0 & 0 & 0 & \frac{12\left(N_{\mathrm{rx}}+N_{\mathrm{tx}}-2\right)}{y^{2}\left(N_{\mathrm{tx}}-1\right)\left(N_{\mathrm{rx}}-1\right)}
\end{array}\right] \\
& \mathrm{CRB}\left(\mathbf{p}^{\mathrm{t}}\right)=\frac{c^{2}}{8 \pi^{2} N_{\mathrm{tx}} \mathrm{SNR}_{1}\left(\beta^{2}+f_{\mathrm{c}}^{2}\right)} \frac{1}{S} \operatorname{diag}\left(\frac{12}{N_{\mathrm{tx}}\left(N_{\mathrm{rx}}-1\right)}, \frac{S}{N_{\mathrm{tx}} N_{\mathrm{rx}}}, \frac{12}{N_{\mathrm{tx}}\left(N_{\mathrm{rx}}-1\right)}\right)
\end{aligned}
$$

TABLE I

MIMO vs. BEAMFORMING COMPARISON

\begin{tabular}{|l|l|l|l|l|}
\hline MIMO/Timed & $N_{\mathrm{rx}}$ & $\gamma[\mathrm{dB}]$ & $\sigma_{\text {thr }}[\mathrm{mV}]$ & $\sigma_{\text {sim }}[\mathrm{mV}]$ \\
\hline MIMO & 4 & -36.9 & 0.062 & 0.022 \\
MIMO & 36 & -32.1 & 0.187 & 0.022 \\
MIMO & 100 & -29.9 & 0.313 & 0.022 \\
Phased & 4 & -33.5 & 0.136 & 0.022 \\
Phased & 36 & -28.7 & 0.406 & 0.022 \\
Phased & 100 & -26.5 & 0.677 & 0.022 \\
\hline
\end{tabular}

By comparing the threshold obtained with the value used in the numerical results, we can demonstrate that we operate at a high SNR regime where the CRB is tight even if a nonmassive array is adopted. To this end, we define the ambiguity probability as $P_{\mathrm{A}}=\frac{1}{2} \operatorname{erfc}\left(\frac{\gamma}{\sqrt{4 \sigma_{n}^{2}}}\right)$ where $\sigma_{n}$ is the noise standard deviation and $\gamma$ is the gap between the main lobe of the $\mathrm{AF}$ and the highest secondary sidelobe [35]. Then, given a certain gap $\gamma$, it is possible to compute the noise threshold as $\sigma_{\mathrm{thr}}=\frac{\gamma}{2} \frac{1}{\operatorname{erfc}^{-1}\left(2 P_{\mathrm{A}}^{*}\right)}$.

In Table $\mathrm{D}$, we report the obtained simulation results. We have considered the Tx moving in a grid of points spaced apart of $0.2 \mathrm{~m}$ in a cube of dimension $8 \times 8 \times 8 \mathrm{~m}^{3}$. The target ambiguity probability has been fixed to $10^{-2}$. The gap $\gamma$ has been set to the minimum side-lobe level considering the three spatial coordinates (that is, to the worst case scenario) and $\sigma_{\text {sim }}$ represents the noise standard deviation used in the numerical results of the paper. As reported in Table I, in all the tested configurations the noise standard deviation used in the numerical results is always much lower than the threshold $\sigma_{\text {thr }}$ above that the ambiguity effect is not anymore negligible, hence meaning that the CRB can be a meaningful metric for the considered scenario.

\section{REFERENCES}

[1] W. H. Chin, Z. Fan, and R. Haines, "Emerging technologies and research challenges for 5G wireless networks," IEEE Wireless Commun., vol. 21, no. 2, pp. 106-112, 2014

[2] F. Rusek et al., "Scaling up MIMO: Opportunities and challenges with very large arrays," IEEE Signal Process. Mag., vol. 30, no. 1, pp. 40-60, 2013.

[3] E. Torkildson, U. Madhow, and M. Rodwell, "Indoor millimeter wave MIMO: Feasibility and performance," IEEE Trans. Wireless Commun. vol. 10 , no. 12 , pp. $4150-4160,2011$.
[4] R. Di Taranto et al., "Location-aware communications for 5G networks: How location information can improve scalability, latency, and robustness of 5G," IEEE Signal Process. Mag., vol. 31, no. 6, pp. 102-112, 2014.

[5] Z. Abu-Shaban et al., "Error bounds for uplink and downlink 3D localization in 5G mmWave systems," arXiv preprint arXiv:1704.03234, 2017.

[6] G. Destino and H. Wymeersch, "On the trade-off between positioning and data rate for mm-wave communication," in Proc. IEEE Int. Conf. on Commun. Workshops (ICCW), May 2017, pp. 797-802.

[7] F. Guidi, A. Guerra, and D. Dardari, "Personal mobile radars with millimeter-wave massive arrays for indoor mapping," IEEE Trans. Mobile Comput., vol. 15, no. 6, pp. 1471-1484, 2016.

[8] F. Guidi et al., "Joint energy detection and massive array design for localization and mapping," IEEE Trans. Wireless Commun., vol. 16, no. 3, pp. 1359-1371, 2017.

[9] K. Witrisal et al., "High-accuracy localization for assisted living: 5G systems will turn multipath channels from foe to friend," IEEE Signal Process. Mag., vol. 33, no. 2, pp. 59-70, 2016.

[10] F. Montorsi, F. Pancaldi, and G. M. Vitetta, "Map-aware models for indoor wireless localization systems: an experimental study," IEEE Trans. Wireless Commun., vol. 13, no. 5, pp. 2850-2862, 2014.

[11] D. Dardari, P. Closas, and P. M. Djurić, "Indoor tracking: Theory, methods, and technologies," IEEE Trans. Veh. Technol., vol. 64, no. 4, pp. 1263-1278, 2015.

[12] N. Garcia et al., "Direct localization for massive MIMO," IEEE Trans. Signal Process., vol. 65, no. 10, pp. 2475-2487, 2017.

[13] W. Hong et al., "Study and prototyping of practically large-scale mmwave antenna systems for 5G cellular devices," IEEE Commun. Mag., vol. 52, no. 9, pp. 63-69, 2014.

[14] Y. Shen and M. Z. Win, "On the accuracy of localization systems using wideband antenna arrays," IEEE Trans. Commun., vol. 58, no. 1, pp. 270-280, 2010

[15] — "Fundamental limits of wideband localizationpart I: A general framework," IEEE Trans. Inf. Theory, vol. 56, no. 10, pp. 4956-4980, 2010.

[16] Y. Han et al., "Performance limits and geometric properties of array localization," IEEE Trans. Inf. Theory, vol. 62, no. 2, pp. 1054-1075, 2016.

[17] T. M. Cover and J. A. Thomas, Elements of information theory. John Wiley \& Sons, 2012

[18] A. J. Weiss, "Direct position determination of narrowband radio frequency transmitters," IEEE Signal Process. Lett., vol. 11, no. 5, pp. 513-516, 2004.

[19] A. Guerra, F. Guidi, and D. Dardari, "On the impact of beamforming strategy on mm-wave localization performance limits," in Proc. IEEE Int. Conf. on Commun. Workshops (ICCW). IEEE, 2017, pp. 809-814.

[20] H. Kaouach et al., "Wideband low-loss linear and circular polarization transmit-arrays in V-band," IEEE Trans. Antennas Propag., vol. 59, no. 7, pp. 2513-2523, 2011.

[21] A. Guerra et al., "A millimeter-wave indoor backscattering channel model for environment mapping," IEEE Trans. Antennas Propag., vol. 65, no. 9, pp. 4935-4940, 2017.

[22] H. Godrich, A. M. Haimovich, and H. V. Poor, "An analysis of phase synchronization mismatch sensitivity for coherent MIMO radar 
systems," in Proc. 3rd IEEE Int. Workshop Comput. Adv. in Multi-Sensor Adaptive Process. (CAMSAP), 2009, pp. 153-156.

[23] A. Guerra, F. Guidi, and D. Dardari, "Position and orientation error bound for wideband massive antenna arrays," in Proc. IEEE Int. Conf. on Commun. Workshop (ICCW), 2015, pp. 853-858.

[24] G. San Antonio, D. R. Fuhrmann, and F. C. Robey, "MIMO radar ambiguity functions," IEEE J. Sel. Topics in Signal Process., vol. 1, no. 1, pp. 167-177, 2007.

[25] J. Hoydis, S. Ten Brink, and M. Debbah, "Massive MIMO in the UL/DL of cellular networks: How many antennas do we need?" IEEE J. Sel. Areas Commun., vol. 31, no. 2, pp. 160-171, 2013.

[26] D. Dardari, E. Falletti, and M. Luise, Satellite and terrestrial radio positioning techniques: a signal processing perspective. Academic Press, 2012.

[27] D. Dardari et al., "Ranging with ultrawide bandwidth signals in multipath environments," Proc. IEEE, vol. 97, no. 2, pp. 404-426, 2009.

[28] J. Li and P. Stoica, "MIMO radar with colocated antennas," IEEE Signal Process. Mag., vol. 24, no. 5, pp. 106-114, 2007.

[29] E. Fishler et al., "Spatial diversity in radars-models and detection performance," IEEE Trans. Signal Process., vol. 54, no. 3, pp. 823-838, 2006.

[30] A. M. Haimovich, R. S. Blum, and L. J. Cimini, "MIMO radar with widely separated antennas," IEEE Signal Process. Mag., vol. 25, no. 1 , pp. 116-129, 2008.

[31] A. F. Molisch, Wireless communications. John Wiley \& Sons, 2007.

[32] Y.-C. Wu, Q. Chaudhari, and E. Serpedin, "Clock synchronization of wireless sensor networks," IEEE Signal Process. Mag., vol. 28, no. 1, pp. 124-138, 2011.

[33] J. Elson, L. Girod, and D. Estrin, "Fine-grained network time synchronization using reference broadcasts," ACM SIGOPS Operating Syst. Rev., vol. 36, no. SI, pp. 147-163, 2002.

[34] K.-L. Noh et al., "Novel clock phase offset and skew estimation using two-way timing message exchanges for wireless sensor networks," IEEE Trans. Commun., vol. 55, no. 4, pp. 766-777, 2007.

[35] H. L. Van Trees, Detection, estimation, and modulation theory. John Wiley \& Sons, 2004.

[36] A. W. Van der Vaart, Asymptotic statistics. Cambridge university press, 2000, vol. 3 .

[37] A. Colombo et al., "Flexible indoor localization and tracking based on a wearable platform and sensor data fusion," IEEE Trans. Instrum. and Meas., vol. 63, no. 4, pp. 864-876, 2014.

[38] P. Moutarlier and R. Chatila, "Stochastic multisensory data fusion for mobile robot location and environment modeling," in 5th Int. Symposium on Robotics Research, vol. 1. Tokyo, 1989.

[39] FCC, "Revision of Part 15 of the Commissions Rules Regarding Operation in the 57-64 GHz Band. August 2013.'

[40] C. Gustafson et al., "On mm-wave multipath clustering and channel modeling," IEEE Trans. Antennas Propag., vol. 62, no. 3, pp. 14451455, 2014.

[41] D. Dardari and F. Guidi, "Direct position estimation from wavefront curvature with single antenna array," in Proc. 8th Int. Conf. on Localization and GNSS 2018. IEEE, 2018.

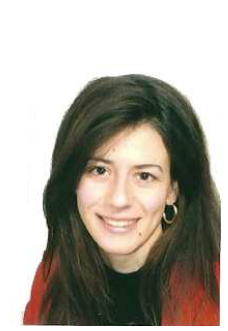

Anna Guerra (S'13 - M'16) received the B.S. and the M.S. degree in electronics and telecommunications engineering from the University of Bologna (UNIBO) in 2009 and 2011, respectively. In 2012, she was a research assistant in a collaboration project between the Consorzio Nazionale Interuniversitario per le Telecomunicazioni (CNIT) and French Atomic Energy Commission (CEA-LETI), Grenoble, France. From mid-2014 to mid-2015, she was a visiting student at CEA-LETI. In 2016, she received the $\mathrm{PhD}$ degree in electronics, telecommunications, and information technologies at UNIBO, Italy. She received the best student paper award at the 2014 IEEE International Conference on Ultra-Wideband in Paris, France. She is recipient of a H2020 IF-GF Marie Skłodowska-Curie fellowship (AirSens project) in collaboration between UNIBO and Stony Brook University, New York, USA. Her research interest includes radio localization and mapping, array signal processing, UWB, and millimeter-wave technology.

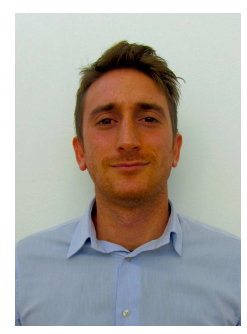

Francesco Guidi (S'09 - M'14) received the B.S degree and the M.S. degree (both summa cum laude) from the University of Bologna in biomedical (2006) and in electronics and telecommunications (2009) engineering, respectively. In 2013 he received the $\mathrm{PhD}$ degree both from Ecole Polytechnique ParisTech, France (Computer Science Specialty) and from University of Bologna, Italy, in electronics, telecommunications and information technologies.

He is currently a Research Fellow at the University of Bologna, Italy. From mid-2013 to mid-2015 he was a postdoctoral researcher at University of Bologna, and from 2015 to 2017 he was the recipient of an individual European Marie Skłodowska-Curie Fellowship at French Atomic Energy Commission (CEA-LETI), Grenoble, France. His research interests include RFID and radar technologies, joint antenna and channel characterization, signal processing, UWB and mm-waves technologies. He was the recipient of the best student paper award at the 2014 IEEE International Conference on Ultra-Wideband. Dr. Guidi serves as reviewer for numerous IEEE Journals, as a reviewer or TPC member for several Conferences, and was a member of the local organization committee for the 2011 IEEE International Conference on Ultra-Wideband.

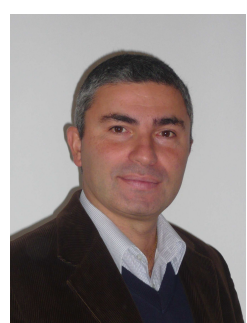

Davide Dardari (M'95 - SM'07) is an Associate Professor at the University of Bologna, Italy. Since 2005, he has been a Research Affiliate at Massachusetts Institute of Technology, USA. His interests are on wireless communications, localization techniques and distributed signal processing. $\mathrm{He}$ published more than 200 technical papers and played several important roles in various National and $\mathrm{Eu}-$ ropean Projects. He received the IEEE Aerospace and Electronic Systems Society's M. Barry Carlton Award (2011) and the IEEE Communications Society Fred W. Ellersick Prize (2012). He is Senior Member of the IEEE where he was the Chair for the Radio Communications Committee of the IEEE Communication Society and Distinguished Lecturer (2018-2019). He was coGeneral Chair of the 2011 IEEE International Conference on Ultra-Wideband and co-organizer of the IEEE International Workshop on Advances in Network Localization and Navigation (ANLN) - ICC 2013-2016 editions. He was also TPC Chair of IEEE International Symposium on Personal, Indoor and Mobile Radio Communications (PIMRC 2018), TPC co-Chair of the Wireless Communications Symposium of the 2007/2017 IEEE International Conference on Communications, and TPC co-Chair of the 2006 IEEE International Conference on Ultra-Wideband. He served as an Editor for IEEE Transactions on Wireless Communications from 2006 to 2012 and as Guest Editor for several Journals. 